Pacific

Journal of

Mathematics

HOMOLOGY MULTIPLIERS AND THE RELATION TYPE OF PARAMETER IDEALS

IAN M. ABERBACH, LAURA GHEZZI AND HuY TÀI HÀ 


\title{
HOMOLOGY MULTIPLIERS AND THE RELATION TYPE OF PARAMETER IDEALS
}

\author{
IAN M. ABERBACH, LAura GHEZZI AND HuY TÀI HÀ
}

\begin{abstract}
The relation type question, raised by $\mathrm{C}$. Huneke, asks whether for a complete equidimensional local ring $R$ there exists a uniform number $N$ such that the relation type of every ideal $I \subset R$ generated by a system of parameters is at most $N$. Wang gave a positive answer to this question when the non-Cohen-Macaulay locus of $R$ (denoted by $\operatorname{NCM}(R)$ ) has dimension zero. In this paper, we first present an example, due to the first author, which gives a negative answer to the question when $\operatorname{dim} \operatorname{NCM}(R) \geq 2$. The major part of our work is to investigate the remaining situation, i.e., when $\operatorname{dim} \operatorname{NCM}(R)=1$. We introduce the notion of homology multipliers and show that the question has a positive answer when $R / \mathscr{A}(R)$ is a domain, where $\mathscr{A}(R)$ is the ideal generated by all homology multipliers in $R$. In a more general context, we also discuss many interesting properties of homology multipliers.
\end{abstract}

\section{Introduction}

Throughout this paper by "ring" we mean a commutative Noetherian ring with identity.

The existence of "uniform bounds" in Noetherian rings is an interesting and important question. By uniform bounds we mean statements which give some numerical bounds not just for one ideal, but for all (or an infinite set of) ideals simultaneously.

In Noetherian rings we have an obvious finiteness condition, i.e., that every ideal is finitely generated; there are, however, deeper forms of finiteness which can be expressed in terms of uniform behavior.

Several types of uniform behavior have been demonstrated recently. See for instance [Huneke 1992; O'Carroll 1987] (uniform Artin-Rees), [Raghavan 1991]

MSC2000: 13A30, 13E15, 13H10.

Keywords: relation type, uniform bound, Rees algebra, Cohen-Macaulay, finiteness.

Aberbach was partially supported by the National Security Agency and the University of Missouri Research Board. Part of this work was done when the authors visited MSRI. We thank the Institute for its financial support. 
(uniform annihilation of local cohomology), and [Lai 1995; Wang 1997a; 1997b] (uniform bounds on relation type). This is by no means a complete list.

In this paper we wish to extend the results of Lai and Wang concerning uniform bounds on relation type of parameter ideals in the papers cited above. We are able to extend the class of rings for which such uniform behavior exists. Moreover, we show that, in general, such uniform behavior should not be expected.

Let $R$ be a Noetherian ring, and let $I=\left(x_{1}, \ldots, x_{n}\right)$ be an ideal of $R$. The Rees algebra $R[I t]$ of $I$ is a quotient of a polynomial ring over $R$. More precisely, there is a canonical surjection $\phi: R\left[T_{1}, \ldots, T_{n}\right] \rightarrow R[I t]$ given by $T_{i} \mapsto x_{i} t$. By giving degree 0 to elements of $R$ and degree 1 to $t$ and $T_{i}$ (for $1 \leq i \leq n$ ), we have that $\phi$ is a homogeneous map, and so the kernel $Q$ of $\phi$ is a homogeneous ideal of $R\left[T_{1}, \ldots, T_{n}\right]$. The relation type of $I$ is defined to be

$$
\operatorname{rt}(I)=\min \left\{k \mid Q_{k}=Q\right\},
$$

where $Q_{k}$ denotes the subideal of $Q$ generated by forms of degrees $\leq k$. The relation type is independent of the choice of the generating set of $I$.

Let $F \in R\left[T_{1}, \ldots, T_{n}\right]$ be a homogeneous form of degree $\delta$. It can be seen that

$$
\phi(F)=F\left(x_{1} t, \ldots, x_{n} t\right)=t^{\delta} F\left(x_{1}, \ldots, x_{n}\right) .
$$

Thus, $F \in Q$ if and only if $F\left(x_{1}, \ldots, x_{n}\right)=0$. Therefore, by saying a relation on $x_{1}, \ldots, x_{n}$ we mean a homogeneous form in $Q$.

An ideal of relation type 1 is said to be of linear type. Huneke [1980, Theorem 3.1] and Valla [1980, Theorem 3.15] proved that if $I$ is generated by a $d$ sequence, then $I$ is of linear type. In particular, an ideal generated by a regular sequence is of linear type. Buchsbaum rings are precisely the rings for which every parameter ideal is of linear type.

If $I$ is generated by a system of parameters (s.o.p.) in the local ring $R$ we say that $I$ is a parameter ideal. The following question was raised by $\mathrm{C}$. Huneke.

Question 1.1 (The relation type question). Let $R$ be a complete equidimensional Noetherian ring of dimension $d$. Does there exist an uniform number $N$ such that for every system of parameters $x_{1}, \ldots, x_{d}$ of $R, \operatorname{rt}\left(x_{1}, \ldots, x_{d}\right) \leq N$ ?

If such a uniform bound exists, we will say that $R$ satisfies bounded relation type, or equivalently, $R$ has a uniform bound on relation type of parameter ideals.

Question 1.1 is closely connected to the strong uniform Artin-Rees property. Let $M \subseteq N$ be two finitely generated $R$-modules. The pair $(M, N)$ is said to have the strong uniform Artin-Rees property if there exists an integer $k$ (depending on $M$ and $N$ ), such that for all $R$-ideals $I$ and all $n \geq k$,

$$
I^{n} M \cap N=I^{n-k}\left(I^{k} M \cap N\right) .
$$


There are several cases in which the strong uniform Artin-Rees property holds [O'Carroll 1987; 1991; Duncan and O'Carroll 1989; Huneke 1992; Planas-Vilanova 2000], but Wang [1997b] has shown that it does not hold in general. See [Planas-Vilanova 2006] for a recent summary and explication of results relating to uniform Artin-Rees theorems.

Being a weaker version of the strong uniform Artin-Rees property [Lai 1995], Question 1.1 has attracted a great deal of attention.

If $R$ is Cohen-Macaulay (CM) any system of parameters forms a regular sequence and so the relation type of any parameter ideal is 1 . CM rings are characterized by the property that the local cohomology modules $H_{\mathfrak{m}}^{i}(R)$ vanish for $i<\operatorname{dim} R$. The next step was to consider local rings $R$ such that $H_{\mathfrak{m}}^{i}(R)$ is finitely generated (therefore of finite length) for all $i<\operatorname{dim} R$. Such rings are called generalized Cohen-Macaulay or rings with finite local cohomology (f.1.c.). Lai showed in [Lai 1995] that bounded relation type holds for rings with finite local cohomology under the assumption that the residue field is finite. In [Wang 1997b] Wang showed that every 2-dimensional Noetherian local ring satisfies bounded relation type. Later he showed in [Wang 1997a] that bounded relation type holds for rings with finite local cohomology without any restriction on the residue field.

The first main result in this paper is to show that bounded relation type does not hold in general. A counterexample, due to the first author, has been known for some time and is presented in Example 2.1. In this example the non-CM locus of $R$ has dimension two (and the counterexample easily generalizes to give counterexamples in rings of arbitrarily high dimensional non-CM locus). On the other hand, as observed at the beginning of Section 6 , if $R$ is a complete equidimensional Noetherian ring, then $R$ has finite local cohomology if and only if $R$ has zero dimensional non-CM locus.

The rest of the paper is devoted to studying the remaining case, i.e., when the non-CM locus of $R$ has dimension one. The methods of [Wang 1997a] cannot be extended to this case. Wang uses strongly in his proof that if $R$ has f.l.c. and $x \in R$ is a parameter, then $R / x R$ has f.l.c., and, moreover, the length of the lower local cohomology modules in $R / x R$ can be bounded in terms of the lengths of the lower local cohomology modules of $R$. When $R$ is not generalized CM then there is no uniform bound on the length of the local cohomology modules of $R / x R$ as $x$ varies among parameters in $R$. Hence, the starting point of our work is an alternative proof of bounded relation type for rings with finite local cohomology which can be generalized. We present this proof in Theorem 6.4. We make use of "homology multipliers", defined in Section 3, and of a "Ramsey number" combinatorial lemma, stated in Section 5.

By a homology multiplier we mean an element in $R$ which annihilates all homology of complexes satisfying the standard rank and height conditions. We denote by 
$\mathscr{A}(R)$ the ideal of $R$ generated by homology multipliers. It follows from a result of Hochster and Huneke that under mild conditions on the ring $R, \mathscr{A}(R)$ is, up to radical, the defining ideal of the non-CM locus in $R$ (see Corollary 3.6). An important property of homology multipliers that we repeatedly use throughout the paper is the fact that, upon multiplying by a homology multiplier colons of monomial ideals in parameters behave as if the parameters were variables (see Remark 3.5). We obtain several interesting results relating relation type and homology multipliers. We show that if an element of an s.o.p. is "adjusted" by a homology multiplier (and results in a new s.o.p.) then the relation type is unchanged (see Theorem 4.3). This result generalizes a result of Lai. Also, the above process does not affect superficiality (see Lemma 4.4).

We show in Section 2 that there are rings where $\operatorname{dim}(R / \mathscr{A}(R)) \geq 2$ which fail to satisfy bounded relation type. Hence our attention is focused on the case that $\operatorname{dim} R / \mathscr{A}(R)=1$.

The new class of rings satisfying bounded relation type is given by this theorem:

Theorem 7.2. Let $(R, \mathfrak{m}, k)$ be a formally unmixed local ring of dimension $d$ such that $\mathscr{A}(\hat{R})$ is a prime ideal of dimension one in the completion $\hat{R}$ of $R$. Then $R$ has a uniform bound on relation type of parameter ideals.

We outline the proof briefly. Our hypotheses allow us to assume that $R$ is complete. Let $I=\left(x_{1}, \ldots, x_{d}\right)$ be a parameter ideal of $R$, and let $\alpha$ be a homology multiplier. Theorem 4.3 shows that if $x_{1}, x_{2}, \ldots, x_{d}+\alpha$ is a system of parameters, then $\operatorname{rt}\left(x_{1}, \ldots, x_{d}\right)=\operatorname{rt}\left(x_{1}, x_{2}, \ldots, x_{d}+\alpha\right)$. This allows us to "modify $x_{d}$ in a convenient way". In particular, we replace $x_{d}$ by $w^{t} x_{d}^{\prime}$ where $w$ is a reduction of $\mathfrak{m} R / \mathscr{A}(R)$ and $x_{d}^{\prime}$ is in a uniformly bounded small power of $\mathfrak{m} R / \mathscr{A}(R)$. We do not have that $H_{\mathfrak{m}}^{i}(R)$ has finite length for all $i<d$ (as in the generalized CM case), but since the ring $R / \mathscr{A}(R)$ is a complete one-dimensional domain we can obtain uniform bounds for the lengths of lower local cohomology modules of $R / x_{d}^{\prime} R$.

Given a relation $F\left(T_{1}, \ldots, T_{d}\right)$ on $x_{1}, \ldots, x_{d-1}, x_{d}=w^{t} x_{d}^{\prime}$ we look at larger and larger "partial sums" and use homology multipliers and uniformly bounded length of local cohomology modules of $R / x_{d}^{\prime} R$ to find a relation $G\left(T_{1}, \ldots, T_{d}\right)$ of uniformly bounded degree such that the initial monomial of $G$ divides the initial monomial of $F$. Inductively, we obtain a bound (which does not depend on the given system of parameters) on the highest degree of a minimal generator of a relation on the system of parameters.

The general argument given is rather subtle, and certainly complicated. However, the basic ideas in the general argument are already present in the argument for two parameters, in which case, the algorithm is transparent. We urge the reader to start with this case by reading Theorem 6.1.

In Corollary 7.4 we apply this theorem just discussed to $F$-pure rings. 


\section{Bounded relation type does not hold in general}

In this section, we will present an example showing that bounded relation type does not hold in general. In our example, the ring has non-CM locus of dimension 2, but it is possible to generalize to rings with non-CM locus of any dimension $\geq 2$.

Example 2.1. Let $R=k \llbracket x, y, z, w \rrbracket$ where $w^{2}=w z=0$. Then, $R$ does not have bounded relation type.

Proof. Let $I_{n}=\left(x^{n-1} y+z^{n}, x^{n}, y^{n}\right)=\left(u_{1}, u_{2}, u_{3}\right)$. Clearly, $I_{n}$ is a parameter ideal for all $n \geq 1$. We will show that for every $n \in \mathbb{N}$, the ideal $I_{n}$ has relation type at least $n$.

We order monomials of $R\left[T_{1}, T_{2}, T_{3}\right]$ using lex and $T_{1}>T_{2}>T_{3}$. Consider the relation $w T_{1}^{n}-w T_{2}^{n-1} T_{3}$ on $u_{1}, u_{2}, u_{3}$ in $R$. Suppose that $w T_{1}^{n}-w T_{2}^{n-1} T_{3}$ can be written as a combination of relations on $u_{1}, u_{2}, u_{3}$ of degree less than $n$. Then there exists a relation $F\left(T_{1}, T_{2}, T_{3}\right)$ on $u_{1}, u_{2}, u_{3}$ of the form

$$
F\left(T_{1}, T_{2}, T_{3}\right)=w T_{1}^{n-1}+\text { smaller terms. }
$$

Let $\bar{F}$ be the image of $F$ in $(R / w R)\left[T_{1}, T_{2}, T_{3}\right]$. Since $\bar{F}$ is a relation on $u_{1}, u_{2}, u_{3}$ in $R / w R$, and $R / w R=k \llbracket x, y, z \rrbracket$ is Cohen-Macaulay, we can write $\bar{F}=\bar{H}_{1} \bar{K}_{1}+$ $\bar{H}_{2} \bar{K}_{2}+\bar{H}_{3} \bar{K}_{3}$, where

$$
K_{1}=-u_{2} T_{1}+u_{1} T_{2}, K_{2}=-u_{3} T_{1}+u_{1} T_{3}, K_{3}=-u_{3} T_{2}+u_{2} T_{3}
$$

are the Koszul relations on $u_{1}, u_{2}, u_{3}$ in $R, \bar{K}_{1}, \bar{K}_{2}, \bar{K}_{3}$ are the Koszul relations on $u_{1}, u_{2}, u_{3}$ in $R / w R$, and $\bar{H}_{1}, \bar{H}_{2}, \bar{H}_{3}$ are polynomials with coefficients in $R / w R$. Hence $F-H_{1} K_{1}-H_{2} K_{2}-H_{3} K_{3} \in(w) R\left[T_{1}, T_{2}, T_{3}\right]$. Write

$$
F-H_{1} K_{1}-H_{2} K_{2}-H_{3} K_{3}=w F^{\prime}
$$

where $F^{\prime}\left(T_{1}, T_{2}, T_{3}\right) \in R\left[T_{1}, T_{2}, T_{3}\right]$. Notice that $F^{\prime}$ contains the term $T_{1}^{n-1}$.

Since $w F^{\prime}\left(u_{1}, u_{2}, u_{3}\right)=0$ in $R$, we have $F^{\prime}\left(u_{1}, u_{2}, u_{3}\right) \in 0:_{R} w=(w, z) R$. Hence the image of $F^{\prime}$ in $(R /(w, z))\left[T_{1}, T_{2}, T_{3}\right]=k \llbracket x, y \rrbracket\left[T_{1}, T_{2}, T_{3} \rrbracket\right.$ is a relation on $x^{n-1} y, x^{n}, y^{n}$ in $k \llbracket x, y \rrbracket$. This implies that

$$
\left(x^{n-1} y\right)^{n-1} \in\left(x^{n}, y^{n}\right)\left(x^{n}, y^{n}, x^{n-1} y\right)^{n-2},
$$

a contradiction (see also [Wang 1997b, Example 6.1]).

Note that the ring $R$ has an embedded prime of dimension two. It would be of interest to find a ring with unbounded relation type which is a domain.

Remark 2.2. Notice that in Example 2.1 the non-CM locus of $R$ is defined by $(z, w)$ and so it has dimension 2 . 
In a similar fashion, we can construct examples of rings with unbounded relation type and non-CM locus of any dimension $\geq 2$. Let $S=k \llbracket t_{1}, \ldots, t_{m}, z, w \rrbracket$ where $w^{2}=w z=0$ and $m \geq 2$. The proof of Example 2.1 shows that

$$
\operatorname{rt}\left(t_{1}^{n-1} t_{2}+z^{n}, t_{1}^{n}, t_{2}^{n}, t_{3}, \ldots, t_{m}\right) \geq n
$$

for every $n \geq 1$. The non-CM locus of $S$ is defined by $(z, w)$ and so it has dimension $m$.

\section{Homology multipliers and superficial sequences}

In this section, we introduce the notion of homology multipliers and investigate a number of properties that will be used later on. We shall also briefly recall the notion of filter-regular and superficial sequences. The proof for some of the results (such as Lemma 3.3 and Corollary 3.4) would be simpler if the ring contains a field. Our arguments work for rings of mixed characteristics as well.

Let $R$ be a Noetherian ring, and let $G$ • be a complex of finitely generated free modules

$$
G_{\bullet}: 0 \rightarrow G_{n} \rightarrow G_{n-1} \rightarrow \cdots \rightarrow G_{i} \rightarrow \cdots \rightarrow G_{1} \rightarrow G_{0} \rightarrow 0 .
$$

Denote by $\alpha_{i}$ the map from $G_{i}$ to $G_{i-1}$. Let $b_{i}$ denote the rank of $G_{i}$, and let $r_{i}=\sum_{t=i}^{n}(-1)^{t-i} b_{i}$ for $1 \leq i \leq n$, while $r_{n+1}=0$. Let $I_{t}\left(\alpha_{i}\right)$ be the ideal generated by the $t \times t$ minors of $\alpha_{i}$.

Recall that a complex $G_{\bullet}$ as above satisfies the standard rank and height conditions if rank $\alpha_{i}=r_{i}$ for $1 \leq i \leq n$, and height $I_{r_{i}}\left(\alpha_{i}\right) \geq i$ whenever $1 \leq i \leq n$ [Hochster and Huneke 1990; 1992]. For simplicity, we denote $I_{r_{i}}\left(\alpha_{i}\right)$ by $I_{i}\left(G_{\bullet}\right)$. If $G_{\bullet}$ is acyclic, then $I_{i}\left(G_{\bullet}\right)$ is an invariant of $H_{0}\left(G_{\bullet}\right)$, and localizes properly.

Definition 3.1. Let $R$ be a Noetherian ring. We say that $z \in R$ is a homology multiplier if for every finite complex $G_{\bullet}$ satisfying the standard rank and height conditions, $z$ annihilates the homology $H_{i}\left(G_{\bullet}\right)$, for every $i \geq 1$. We denote by $\mathscr{A}(R)$ the ideal of $R$ generated by homology multipliers.

The notion of homology multipliers is a generalization of Cohen-Macaulay multipliers [Hochster and Huneke 1990; 1992]. Recall that $R$ is equidimensional if $\operatorname{dim} R / p=\operatorname{dim} R$ for all minimal prime ideals of $R$. The following result is due to Hochster and Huneke.

Theorem 3.2 [Hochster and Huneke 1990, Theorem 11.8]. Let $R$ be an equidimensional local ring which is a homomorphic image of a Gorenstein ring. Let $z$ be an element of $R$ such that $R_{z}$ is Cohen-Macaulay. Then $z$ has a fixed power $z^{\prime}$ such that $z^{\prime}$ is a homology multiplier.

Homology multipliers are introduced to handle colons of monomial ideals in parameters (see Remark 3.5 for a precise statement). To see this, we need: 


\section{Lemma 3.3. Let}

$$
R=\mathbb{Z}\left[Y_{1}, Y_{1}^{-1}, \ldots, Y_{t}, Y_{t}^{-1}\right]\left[X_{1}, \ldots, X_{d}\right],
$$

where $Y_{1}, \ldots, Y_{t}, X_{1}, \ldots, X_{d}$ are variables. Let I be an ideal generated by monomials in $X_{1}, \ldots, X_{d}$.

(1) There exists a resolution $F_{\bullet}$ of $R / I$ of length $\leq d$. Furthermore, for every $1 \leq i \leq d$,

$$
\prod_{1 \leq j_{1}<\cdots<j_{i} \leq d}\left(X_{j_{1}}, \ldots, X_{j_{i}}\right) \subseteq \sqrt{I_{i}\left(F_{\bullet}\right)} .
$$

(2) As a consequence, (3-1) holds for every resolution $F_{\bullet}$ of $R / I$.

Proof. First we show the existence of a resolution of $R / I$ of length $\leq d$. This is clear if $d=1$, so we can assume that $d>1$. We use induction on the number of generators $\mu(I)$ of $I$. The claim is trivial if $\mu(I)=1$. Assume that $I=\left(I^{\prime}, m\right)$ where $m$ is a monomial with the largest possible exponent of $X_{d}$, and $I^{\prime}$ is a monomial ideal with $\mu\left(I^{\prime}\right) \geq 1$. Then $I^{\prime}: m$ is a monomial ideal in $X_{1}, \ldots, X_{d-1}$. We have a short exact sequence

$$
0 \rightarrow R / m\left(I^{\prime}: m\right) \rightarrow R / I^{\prime} \oplus R / m R \rightarrow R / I \rightarrow 0 .
$$

By induction on $\mu(I)$ we have that $R / I^{\prime} \oplus R / m R$ has a resolution of length $\leq d$. By induction on $d$ we see that $R / m\left(I^{\prime}: m\right)$ has a resolution of length $\leq d-1$. Hence the mapping cone is a resolution of $R / I$ of length $\leq d$. In particular this shows that if $G_{\bullet}$ is any resolution of $R / I$, then $I_{d+1}\left(G_{\bullet}\right)=R$.

Next we show that $\left(X_{1}, \ldots, X_{d}\right) \subseteq \sqrt{I_{d}\left(F_{\bullet}\right)}$. This is clear if $I_{d}\left(F_{\bullet}\right)=R$, so we can assume that $I_{d}\left(F_{\bullet}\right)$ is a proper ideal. If there exists $i$, with $1 \leq i \leq d$, such that $X_{i} \notin \sqrt{I_{d}\left(F_{\bullet}\right)}$, then localizing at $X_{i}$ we obtain a contradiction to the existence of a resolution of length $\leq d-1$ in the ring

$$
\mathbb{Z}\left[Y_{1}, Y_{1}^{-1}, \ldots, Y_{t}, Y_{t}^{-1}, X_{i}, X_{i}^{-1}\right]\left[X_{1}, \ldots, X_{i-1}, X_{i+1}, \ldots, X_{d}\right] .
$$

Now let $1 \leq i \leq d-1$ and let $1 \leq j_{1}<\cdots<j_{i} \leq d-1$. Localize at the $d-i$ variables $\left\{X_{1}, \ldots, X_{d}\right\} \backslash\left\{X_{j_{1}}, \ldots, X_{j_{i}}\right\}$ and conclude as above. This finishes the proof of (1).

Part (2) follows form (1) since the ideals $I_{i}\left(F_{\bullet}\right)$ do not depend on the resolution of $R / I$.

Corollary 3.4. Let $R$ be an equidimensional catenary local ring of dimension $d$ and let $x_{1}, \ldots, x_{d}$ be a system of parameters of $R$. Let $\phi: \mathbb{Z}\left[X_{1}, \ldots, X_{d}\right] \rightarrow R$ be the ring homomorphism from a polynomial ring over $\mathbb{Z}$ to $R$ sending $X_{i}$ to $x_{i}$ for all $i$. Suppose $J$ is a monomial ideal in $\mathbb{Z}\left[X_{1}, \ldots, X_{d}\right]$ and $G_{\bullet}$ is a resolution 
of $\mathbb{Z}\left[X_{1}, \ldots, X_{d}\right] / J$ of length at most $d$. Let $F_{\bullet}=G_{\bullet} \otimes R$ be the complex obtained from $G_{\bullet}$ by base change. Then, $F_{\bullet}$ satisfies the standard rank and height conditions.

Proof. By Lemma 3.3, for $1 \leq i \leq d$,

$$
\prod_{1 \leq j_{1}<\cdots<j_{i} \leq d}\left(X_{j_{1}}, \ldots, X_{j_{i}}\right) \subseteq \sqrt{I_{i}\left(G_{\bullet}\right)} .
$$

This implies that $\prod_{1 \leq j_{1}<\cdots<j_{i} \leq d}\left(x_{j_{1}}, \ldots, x_{j_{i}}\right) \subseteq \sqrt{I_{i}\left(F_{\bullet}\right)}$. Since $F_{\bullet}$ is obtained by tensoring $G_{\bullet}$ with $R$, the $r_{i}$ 's are unchanged. Also, since $R$ is equidimensional and catenary, we have

$$
\text { height } \prod_{1 \leq j_{1}<\cdots<j_{i} \leq d}\left(x_{j_{1}}, \ldots, x_{j_{i}}\right) \geq i .
$$

Hence, $F_{\bullet}$ satisfies the standard rank and height conditions since $G_{\bullet}$ certainly does.

The following remark will be used very often in the rest of the paper.

Remark 3.5. Let $R$ be an equidimensional catenary local ring of dimension $d$ and let $x_{1}, \ldots, x_{d}$ be a system of parameters of $R$. Let $S=\mathbb{Z}\left[X_{1}, \ldots, X_{d}\right]$ be a polynomial ring over $\mathbb{Z}$, and let $\phi$ be as in Corollary 3.4. For a $d$-uple of nonnegative integers $\boldsymbol{n}=\left(n_{1}, \ldots, n_{d}\right)$, we denote by $\boldsymbol{X}^{\boldsymbol{n}}$ the monomial $X_{1}^{n_{1}} \ldots X_{d}^{n_{d}}$, and by $\boldsymbol{x}^{\boldsymbol{n}}$ the monomial $x_{1}^{n_{1}} \ldots x_{d}^{n_{d}}$. Let $I=\left(\boldsymbol{X}^{\boldsymbol{n}_{1}}, \ldots, \boldsymbol{X}^{\boldsymbol{n}_{t}}\right)$ and let $\boldsymbol{X}^{\boldsymbol{m}}$ be any monomial in $S$ such that $\boldsymbol{X}^{\boldsymbol{m}} \notin I$. By considering the minimal free resolution of $I+\left(X^{\boldsymbol{m}}\right)$,

$$
\cdots \rightarrow S^{l} \stackrel{\partial_{2}}{\rightarrow} S^{t+1} \rightarrow S \rightarrow \frac{S}{I+\left(\boldsymbol{X}^{\boldsymbol{m}}\right)} \rightarrow 0,
$$

it can be seen that the colon ideal $I:\left(\boldsymbol{X}^{\boldsymbol{m}}\right)$ is generated by elements of the last row of the matrix of $\partial_{2}$. Thus, as a consequence of Corollary 3.4, for any homology multiplier $z \in R$, we have

$$
z\left(I R:_{R} \boldsymbol{x}^{\boldsymbol{m}}\right)=\left(I:_{S} \boldsymbol{X}^{\boldsymbol{m}}\right) R .
$$

More generally, if $J=\left(\boldsymbol{X}^{\boldsymbol{m}_{1}}, \ldots, \boldsymbol{X}^{\boldsymbol{m}_{s}}\right)$ is another monomial ideal in $S$, and $\mathfrak{a}=I R=\left(\boldsymbol{x}^{n_{1}}, \ldots, \boldsymbol{x}^{n_{t}}\right)$ and $\mathfrak{b}=J R=\left(\boldsymbol{x}^{\boldsymbol{m}_{1}}, \ldots, \boldsymbol{x}^{\boldsymbol{m}_{s}}\right)$, then $z\left(\mathfrak{a}::_{R} \mathfrak{b}\right)=\left(I:_{S}\right.$ $J) R$. Hence, up to multiplying by a homology multiplier, colons of monomials in $x_{1}, \ldots, x_{d}$ behave as if the elements $x_{1}, \ldots, x_{d}$ were variables.

Let $R$ be an equidimensional catenary local ring with maximal ideal $\mathfrak{m}$ and let $z \in \mathscr{A}(R)$. Let $x_{1}, \ldots, x_{k}$ be a sequence of elements of $\mathfrak{m}$ that is part of a system of parameters of $R$. Then $z$ annihilates all the higher Koszul homology $H_{i}\left(x_{1}, \ldots, x_{k}, R\right), i \geq 1$. In particular,

$$
z\left(\left(x_{1}, \ldots, x_{k-1}\right) R:_{R} x_{k} R\right) \subseteq\left(x_{1}, \ldots, x_{k-1}\right) R
$$


that is, $z$ is a Cohen-Macaulay multiplier [Hochster and Huneke 1990; 1992].

The next corollary shows that up to radical, $\mathscr{A}(R)$ is the defining ideal of the non-Cohen-Macaulay locus in $R$.

Corollary 3.6. Let $R$ be an equidimensional local ring which is a homomorphic image of a Gorenstein ring. Let $z \in R$. Then $z \in \sqrt{\mathscr{A}(R)}$ if and only if $R_{z}$ is Cohen-Macaulay.

Proof. One direction is Theorem 3.2. Conversely, let $z \in \sqrt{\mathscr{A}(R)}$, and let $x_{1} / 1, \ldots$, $x_{n} / 1$ be a system of parameters of $R_{p}$, where $p$ is a prime ideal of $R$ such that $z \notin p$. There exists a power $z^{\prime}$ of $z$ such that $z^{\prime}\left(\left(x_{1}, \ldots, x_{k-1}\right) R:_{R} x_{k} R\right) \subseteq\left(x_{1}, \ldots, x_{k-1}\right) R$ for every $1 \leq k \leq n$. Hence $x_{1} / 1, \ldots, x_{n} / 1$ form a regular sequence in $R_{p}$.

We conclude this section recalling two definitions that we will use later on.

Definition 3.7. Let $S$ be a standard $\mathbb{N}$-graded algebra over a local ring $S_{0}$ (i.e., $\left.S=S_{0}\left[S_{1}\right]\right)$. The sequence of elements $z_{1}, \ldots, z_{n} \in S$ is called filter-regular if, for each $i \geq 1,\left(\left(z_{1}, \ldots, z_{i-1}\right): z_{i}\right)_{n}=\left(z_{1}, \ldots, z_{i-1}\right)_{n}$ for $n \gg 0$.

When $S_{0}$ has infinite residue field, any homogeneous ideal of $S$ may be generated by a filter-regular sequence, by [Trung 1987, Lemma 3.1]. If $z_{1}, \ldots, z_{n}$ is a filter-regular sequence, the Koszul homology modules $H_{i}\left(z_{1}, \ldots, z_{n} ; S\right)$ vanish in sufficiently high degree; see [Aberbach and Huneke 1993, Lemma 4.7].

We will be most interested in the case where $S$ is the associated graded ring of an ideal generated by a system of parameters.

Definition 3.8. Let $(R, \mathfrak{m})$ be a local ring with infinite residue field and let $I$ be an ideal. Recall that $x \in I$ is a superficial element for $I$ if for some integer $c$ and all $n \gg 0,\left(I^{n}: x\right) \cap I^{c}=I^{n-1}$. Let $S=G(I)$, the associated graded ring of $I$. Let $x \in I$ and let $z=x+I^{2} \in S_{1}$. Notice that $x$ is a superficial element for $I$ if and only if $z$ is a filter-regular element. Let $I=\left(x_{1}, \ldots, x_{d}\right)$ and let $z_{i}=x_{i}+I^{2} \in S_{1}$ for $1 \leq i \leq d$. If the sequence $z_{1}, \ldots, z_{d}$ is filter-regular, we say that the sequence $x_{1}, \ldots, x_{d}$ is a superficial sequence for $I$.

Remark 3.9. If $x_{1}, \ldots, x_{d}$ is a superficial sequence for $I$ then there exists an integer $c^{\prime}$ such that, if $1 \leq i \leq d$ and $r_{1} x_{1}+\cdots+r_{i} x_{i} \in I^{n}$ with $n \geq c^{\prime}$ and $r_{1}, \ldots, r_{i} \in I^{c^{\prime}}$ then we have $r_{1} x_{1}+\cdots+r_{i} x_{i}=r_{1}^{\prime} x_{1}+\cdots+r_{i}^{\prime} x_{i}$ with $r_{1}^{\prime}, \ldots, r_{i}^{\prime} \in I^{n-1}$. Notice that the converse is not true in general.

Given any set of generators $a_{1}, \ldots, a_{d}$ for $I$, there is a Zariski-open set $U$ of $(R / \mathfrak{m})^{d^{2}}$ such that setting $x_{i}=\sum_{j=1}^{d} u_{i j} a_{j}$ with $\left(\overline{u_{i j}}\right)_{1 \leq i, j \leq d} \in U$, where $\overline{u_{i j}}$ is the image of $u_{i j}$ in $R / \mathfrak{m}$, gives a superficial sequence generating $I$. 


\section{An application of homology multipliers to relation type}

In this section, we shall investigate two interesting properties of homology multipliers. First, we show that when an element of a system of parameters is changed by a homology multiplier (such that we still have an s.o.p.), the relation type is not changed (see Theorem 4.3). This provides a nice tool for studying relation type by modifying s.o.p.'s in a "convenient" way, which we will apply in Sections 6 and 7. Secondly, we prove that superficiality is also preserved by changing an element of an s.o.p. by a homology multiplier (see Lemma 4.4).

Throughout this section, $R$ is an equidimensional catenary local ring of dimension $d$ and $\left\langle x_{1}, \ldots, x_{d}\right\rangle$ denotes an s.o.p. of $R$.

For a tuple $\boldsymbol{n}=\left(n_{1}, \ldots, n_{d}\right)$, let $|\boldsymbol{n}|=n_{1}+\cdots+n_{d}$ and let $\boldsymbol{x}^{\boldsymbol{n}}$ denote $x_{1}^{n_{1}} \cdots x_{d}^{n_{d}}$. From now on, we shall always use graded reverse lex monomial ordering. Suppose

$$
F\left(T_{1}, \ldots, T_{d}\right)=\sum_{|\boldsymbol{n}|=n, \boldsymbol{n} \leq \boldsymbol{n}_{0}} r_{\boldsymbol{n}} \boldsymbol{T}^{\boldsymbol{n}}
$$

is a homogeneous form of degree $n$ in $R\left[T_{1}, \ldots, T_{d}\right]$ with leading term $r_{n_{0}} \boldsymbol{T}^{\boldsymbol{n}_{0}}$ which provides a relation on $x_{1}, \ldots, x_{d}$, i.e. $F\left(x_{1}, \ldots, x_{d}\right)=0$ (if a monomial $\boldsymbol{T}^{\boldsymbol{n}}$ does not appear in $F$, we shall take $r_{n}=0$ ). Let $A$ be a new variable and write

$$
F\left(T_{1}, \ldots, T_{d}\right)=F\left(T_{1}+A, T_{2}, \ldots, T_{d}\right)-A G\left(T_{1}+A, T_{2}, \ldots, T_{d}\right) .
$$

Lemma 4.1. Let $R$ be an equidimensional catenary local ring of dimension $d$. Suppose $\boldsymbol{x}=\left\langle x_{1}, \ldots, x_{d}\right\rangle$ and $\boldsymbol{y}=\left\langle y_{1}, x_{2}, \ldots, x_{d}\right\rangle$ are s.o.p.'s such that $\alpha=$ $y_{1}-x_{1} \in \mathscr{A}(R)$ is a homology multiplier. Then using the notation as above,

$$
\alpha G\left(y_{1}, x_{2}, \ldots, x_{d}\right) \in\left(\boldsymbol{y}^{\boldsymbol{m}} \mid \boldsymbol{m}<\boldsymbol{n}_{0}\right) .
$$

Proof. Let $J_{T}=\left(\boldsymbol{T}^{\boldsymbol{n}}|| \boldsymbol{n} \mid=n, \boldsymbol{n} \leq \boldsymbol{n}_{0}\right) \subseteq \mathbb{Z}\left[T_{1}, \ldots, T_{d}\right]$ and $J=\left(\boldsymbol{x}^{\boldsymbol{n}}|| \boldsymbol{n} \mid=\right.$ $\left.n, \boldsymbol{n} \leq \boldsymbol{n}_{0}\right)=J_{T} \otimes R \subseteq R$. Then, $J_{T}$ is a stable ideal in the sense of [Eliahou and Kervaire 1990] if we reverse the order of variables, i.e., if we list the variables as $T_{d}, \ldots, T_{1}$, and so it admits an Eliahou-Kervaire graded free resolution. Notice that [Eliahou and Kervaire 1990] in fact provided a graded free resolution of $J_{T}$ rather than of $\mathbb{Z}\left[T_{1}, \ldots, T_{d}\right] / J_{T}$; there is, therefore, a shift of one index in our resolution compared to that given in [Eliahou and Kervaire 1990]. By base change, $R / J$ admits the following complex

$$
\boldsymbol{F}_{\bullet}: \cdots \rightarrow F_{2} \stackrel{\partial_{2}}{\rightarrow} F_{1} \stackrel{\partial_{1}}{\rightarrow} R \rightarrow R / J \rightarrow 0
$$

where $F_{i}$ 's are free $R$-modules, and $\partial_{2}$ is given by a matrix $M=M(F)$ where each column has exactly 2 nonzero entries and is of the form

$$
\left[0 \cdots-x_{j} \cdots x_{i} \cdots 0\right]^{\mathrm{T}} \text { for some } i<j .
$$


It follows from Corollary 3.4 that $\boldsymbol{F}$ • satisfies the standard rank and height conditions. Since $F\left(x_{1}, \ldots, x_{d}\right)=0$, we have

$$
\left[r_{n_{0}} \cdots r_{\boldsymbol{n}} \cdots\right]^{\mathrm{T}} \in \operatorname{ker} \partial_{1},
$$

where $\left[r_{n_{0}} \cdots r_{\boldsymbol{n}} \cdots\right]^{\mathrm{T}}$ is the column vector of coefficients in $F$. Since $\alpha$ annihilates $H_{1}\left(\boldsymbol{F}_{\bullet}\right)$, we have

$$
\alpha\left[r_{n_{0}} \cdots r_{\boldsymbol{n}} \cdots\right]^{\mathrm{T}} \in \operatorname{im} \partial_{2} .
$$

Let $K=K(F)$ be the number of columns of $M$ and let $C_{1}, \ldots, C_{K}$ be the columns of $M$. We then can write

$$
\alpha\left[r_{n_{0}} \cdots r_{n} \cdots\right]^{\mathrm{T}}=b_{1} C_{1}+\cdots+b_{K} C_{K}
$$

where $b_{1}, \ldots, b_{K} \in R$.

Consider an arbitrary term $r_{\boldsymbol{n}} \boldsymbol{T}^{\boldsymbol{n}}=r_{\boldsymbol{n}} T_{1}^{n_{1}} \ldots T_{d}^{n_{d}}$ of $F\left(T_{1}, \ldots, T_{d}\right)$. We have

$$
\begin{aligned}
r_{\boldsymbol{n}} \boldsymbol{T}^{\boldsymbol{n}} & =r_{\boldsymbol{n}}\left(T_{1}+A-A\right)^{n_{1}} T_{2}^{n_{2}} \ldots T_{d}^{n_{d}} \\
& =r_{\boldsymbol{n}}\left(T_{1}+A\right)^{n_{1}} T_{2}^{n_{2}} \ldots T_{d}^{n_{d}}+r_{\boldsymbol{n}} T_{2}^{n_{2}} \ldots T_{d}^{n_{d}}\left(\sum_{l=1}^{n_{1}}(-1)^{l}\left(\begin{array}{c}
n_{1} \\
l
\end{array}\right)\left(T_{1}+A\right)^{n_{1}-l} A^{l}\right) \\
& =r_{\boldsymbol{n}}\left(T_{1}+A\right)^{n_{1}} T_{2}^{n_{2}} \ldots T_{d}^{n_{d}}+A r_{\boldsymbol{n}} \frac{\boldsymbol{T}^{\boldsymbol{n}}}{T_{1}^{n_{1}}}\left(\sum_{l=1}^{n_{1}}(-1)^{l}\left(\begin{array}{c}
n_{1} \\
l
\end{array}\right)\left(T_{1}+A\right)^{n_{1}-l} A^{l-1}\right) .
\end{aligned}
$$

Thus, we have

$$
A G\left(T_{1}+A, T_{2}, \ldots, T_{d}\right)=\sum_{\substack{r_{n} \boldsymbol{T}^{n} \text { is a term in } \\
F\left(T_{1}, \ldots, T_{d}\right)}} A r_{n} \frac{\boldsymbol{T}^{\boldsymbol{n}}}{T_{1}^{n_{1}}}\left(\sum_{l=1}^{n_{1}}(-1)^{l}\left(\begin{array}{c}
n_{1} \\
l
\end{array}\right)\left(T_{1}+A\right)^{n_{1}-l} A^{l-1}\right) .
$$

This implies that

$$
\alpha G\left(y_{1}, x_{2}, \ldots, x_{d}\right)=\sum_{\substack{r_{n} x^{n} \text { is a term in } \\
F\left(x_{1}, \ldots, x_{d}\right)}} \alpha r_{n} \frac{\boldsymbol{x}^{n}}{x_{1}^{n_{1}}}\left(\sum_{l=1}^{n_{1}}(-1)^{l}\left(\begin{array}{c}
n_{1} \\
l
\end{array}\right)\left(x_{1}+\alpha\right)^{n_{1}-l} \alpha^{l-1}\right) .
$$

It follows from (4-2) that $\alpha r_{n} \in\left(b_{1}, \ldots, b_{K}\right) R$. Hence, $\alpha G\left(y_{1}, x_{2}, \ldots, x_{d}\right)$ can be written as a combination of $b_{1}, \ldots, b_{K}$ with coefficients in $R$.

Let $\boldsymbol{e}_{l}$ be the $l$-th unit vector of $\mathbb{N}^{d}$ and suppose $C_{1}=\left[\begin{array}{lllllll}0 & \cdots & -x_{j} & \cdots & x_{i} & \cdots & 0\end{array}\right]^{\mathrm{T}}$ for some fixed $i<j$. Now, consider the contribution to $\alpha G\left(y_{1}, x_{2}, \ldots, x_{d}\right)$ coming from $b_{1} C_{1}$ after substituting (4-2) to (4-3). It follows from (4-2) that this contribution results from $r_{\boldsymbol{m}+\boldsymbol{e}_{i}} \boldsymbol{x}^{\boldsymbol{m}+\boldsymbol{e}_{i}}-r_{\boldsymbol{m}+\boldsymbol{e}_{j}} \boldsymbol{x}^{\boldsymbol{m}+\boldsymbol{e}_{j}}$ of $F\left(x_{1}, \ldots, x_{d}\right)$, where $\boldsymbol{e}_{i}>\boldsymbol{e}_{j}$. If $i>1$, in which case $j>i>1$, then in (4-3), terms coming from $r_{\boldsymbol{m}+\boldsymbol{e}_{i}} \boldsymbol{x}^{\boldsymbol{m}+\boldsymbol{e}_{i}}$ and 
$r_{\boldsymbol{m}+\boldsymbol{e}_{j}} \boldsymbol{x}^{\boldsymbol{m}+\boldsymbol{e}_{j}}$ cancel each other since they have the same power of $x_{1}$. Suppose that $j>i=1$. Substituting (4-2) to (4-3), $r_{\boldsymbol{m}+\boldsymbol{e}_{1}} \boldsymbol{x}^{\boldsymbol{m}+\boldsymbol{e}_{1}}=r_{\boldsymbol{m}+\boldsymbol{e}_{1}} x_{1}^{m_{1}+1} x_{2}^{m_{2}} \ldots x_{d}^{m_{d}}$ gives

$$
-b_{1} x_{j} \frac{\boldsymbol{x}^{\boldsymbol{m}}}{x_{1}^{m_{1}}}\left(\sum_{l=1}^{m_{1}+1}(-1)^{l}\left(\begin{array}{c}
m_{1}+1 \\
l
\end{array}\right)\left(x_{1}+\alpha\right)^{m_{1}+1-l} \alpha^{l-1}\right)
$$

and $r_{\boldsymbol{m}+\boldsymbol{e}_{j}} \boldsymbol{x}^{\boldsymbol{m}+\boldsymbol{e}_{j}}$ gives

$$
b_{1} x_{1} \frac{\boldsymbol{x}^{\boldsymbol{m}} x_{j}}{x_{1}^{m_{1}}}\left(\sum_{l=1}^{m_{1}}(-1)^{l}\left(\begin{array}{c}
m_{1} \\
l
\end{array}\right)\left(x_{l}+\alpha\right)^{m_{1}-l} \alpha^{l-1}\right) .
$$
Thus these two terms of $F\left(x_{1}, \ldots, x_{d}\right)$ contribute $b_{1} \frac{x^{m} x_{j}}{x_{1}^{m_{1}}} S$ to $\alpha G\left(y_{1}, x_{2}, \ldots, x_{d}\right)$,
where

$$
S:=\sum_{l=1}^{m_{1}}(-1)^{l}\left(\begin{array}{c}
m_{1} \\
l
\end{array}\right)\left(x_{1}+\alpha\right)^{m_{1}-l} \alpha^{l-1} x_{1}-\sum_{l=1}^{m_{1}+1}(-1)^{l}\left(\begin{array}{c}
m_{1}+1 \\
l
\end{array}\right)\left(x_{1}+\alpha\right)^{m_{1}+1-l} \alpha^{l-1} .
$$

Note that $\left(\begin{array}{c}m_{1}+1 \\ l\end{array}\right)=\left(\begin{array}{c}m_{1} \\ l\end{array}\right)+\left(\begin{array}{c}m_{1} \\ l-1\end{array}\right)$. Therefore,

$$
\begin{array}{r}
S=\sum_{l=1}^{m_{1}}(-1)^{l}\left(\begin{array}{c}
m_{1} \\
l
\end{array}\right)\left(x_{1}+\alpha\right)^{m_{1}-l} \alpha^{l-1} x_{1}-\sum_{l=1}^{m_{1}+1}(-1)^{l}\left(\begin{array}{c}
m_{1} \\
l
\end{array}\right)\left(x_{1}+\alpha\right)^{m_{1}+1-l} \alpha^{l-1} \\
-\sum_{l=1}^{m_{1}+1}(-1)^{l}\left(\begin{array}{c}
m_{1} \\
l-1
\end{array}\right)\left(x_{1}+\alpha\right)^{m_{1}+1-l} \alpha^{l-1} \\
=\sum_{l=1}^{m_{1}}(-1)^{l}\left(\begin{array}{c}
m_{1} \\
l
\end{array}\right)\left(x_{1}+\alpha\right)^{m_{1}-l} \alpha^{l-1} x_{1}-\sum_{l=1}^{m_{1}+1}(-1)^{l}\left(\begin{array}{c}
m_{1} \\
l
\end{array}\right)\left(x_{1}+\alpha\right)^{m_{1}+1-l} \alpha^{l-1} \\
\quad+\sum_{l=0}^{m_{1}}(-1)^{l}\left(\begin{array}{c}
m_{1} \\
l
\end{array}\right)\left(x_{1}+\alpha\right)^{m_{1}-l} \alpha^{l} \\
=\sum_{l=1}^{m_{1}}(-1)^{l}\left(\begin{array}{c}
m_{1} \\
l
\end{array}\right)\left(\left(x_{1}+\alpha\right)^{m_{1}-l} \alpha^{l-1} x_{1}+\left(x_{1}+\alpha\right)^{m_{1}-l} \alpha^{l}\right)+\left(x_{1}+\alpha\right)^{m_{1}} \\
\quad-\sum_{l=1}^{m_{1}+1}(-1)^{l}\left(\begin{array}{c}
m_{1} \\
l
\end{array}\right)\left(x_{1}+\alpha\right)^{m_{1}+1-l} \alpha^{l-1} .
\end{array}
$$

We eventually get

$$
\begin{gathered}
S=\sum_{l=1}^{m_{1}+1}(-1)^{l}\left(\begin{array}{c}
m_{1} \\
l
\end{array}\right)\left(x_{1}+\alpha\right)^{m_{1}+1-l} \alpha^{l-1} \\
-\sum_{l=1}^{m_{1}+1}(-1)^{l}\left(\begin{array}{c}
m_{1} \\
l
\end{array}\right)\left(x_{1}+\alpha\right)^{m_{1}+1-l} \alpha^{l-1}+\left(x_{1}+\alpha\right)^{m_{1}} \\
=\left(x_{1}+\alpha\right)^{m_{1}} .
\end{gathered}
$$

Hence, in $\alpha G\left(y_{1}, x_{1}, \ldots, x_{d}\right)$, the term with factor $b_{1}$ is $b_{1} \boldsymbol{y}^{\boldsymbol{m}+\boldsymbol{e}_{j}}$. Since $\boldsymbol{y}^{\boldsymbol{m}+\boldsymbol{e}_{j}}<$ $\boldsymbol{y}^{\boldsymbol{n}_{0}}$, we have $b_{1} \boldsymbol{y}^{\boldsymbol{m}+\boldsymbol{e}_{j}} \in\left(\boldsymbol{y}^{\boldsymbol{m}} \mid \boldsymbol{m}<\boldsymbol{n}_{0}\right)$. A similar analysis works for the contribution to $\alpha G\left(y_{1}, x_{2}, \ldots, x_{d}\right)$ coming from $b_{2} C_{2}, \ldots, b_{K} C_{K}$. 
Suppose $\boldsymbol{x}=\left\langle x_{1}, \ldots, x_{d}\right\rangle$ and $\boldsymbol{y}=\left\langle y_{1}, x_{2}, \ldots, x_{d}\right\rangle$ are s.o.p.'s such that $y_{1}-x_{1}=$ $\alpha \in \mathscr{A}(R)$. As before, let $F\left(T_{1}, \ldots, T_{d}\right)$ be a homogeneous form of degree $N$ with leading term $r_{n_{0}} \boldsymbol{T}^{\boldsymbol{n}_{0}}$ that gives a relation on $\boldsymbol{x}$. Let $M=M(F), K=K(F)$ and $C_{1}, \ldots, C_{K}$ be as in Lemma 4.1. Suppose $C_{j_{1}}, \ldots, C_{j_{s}}$ are the columns that contain $x_{1}$, i.e. for $i \leq l \leq s, C_{j_{l}}$ has the form

$$
C_{j_{l}}=\left[\begin{array}{lllllll}
0 & \cdots & -x_{j l} & \cdots & x_{1} & \cdots & 0
\end{array}\right]^{\mathrm{T}} .
$$

Suppose that in (4-2), $\alpha r_{n_{l}}$, for each $l=1, \ldots, s$, lie in the same row as $x_{1}$. It follows from the proof of Lemma 4.1 that, for each choice of $b_{1}, \ldots, b_{K}$ in (4-2), we can write

$$
0=F\left(x_{1}, \ldots, x_{d}\right)=F\left(y_{1}, x_{2}, \ldots, x_{d}\right)+\sum_{l=1}^{s} b_{j_{l}} y^{\boldsymbol{n}_{l}}
$$

Set

$$
\tilde{F}_{b_{1}, \ldots, b_{K}}\left(T_{1}, \ldots, T_{d}\right)=F\left(T_{1}, \ldots, T_{d}\right)+\sum_{l=1}^{s} b_{j_{l}} \boldsymbol{T}^{\boldsymbol{n}_{l}} .
$$

Then, for each choice of $b_{1}, \ldots, b_{K}$ in (4-2), $\tilde{F}_{b_{1}, \ldots, b_{K}}\left(T_{1}, \ldots, T_{d}\right)$ provides a relation on $\boldsymbol{y}$. Notice that for any $b_{1}, \ldots, b_{K}, \tilde{F}_{b_{1}, \ldots, b_{K}}\left(T_{1}, \ldots, T_{d}\right)$ and $F\left(T_{1}, \ldots, T_{d}\right)$ always have the same leading term. We shall denote by $\Phi_{\alpha, b_{1}, \ldots, b_{K}}$ the function which sends a relation $F\left(T_{1}, \ldots, T_{d}\right)$ on $\boldsymbol{x}=\left\langle x_{1}, \ldots, x_{d}\right\rangle$ to the relation $\tilde{F}_{b_{1}, \ldots, b_{K}}\left(T_{1}, \ldots, T_{d}\right)$ on $\boldsymbol{y}=\left\langle y_{1}, x_{2}, \ldots, x_{d}\right\rangle$.

Lemma 4.2 (compare [Lai 1995, Theorem 3.6]). Let $\boldsymbol{x}=\left\langle x_{1}, \ldots, x_{d}\right\rangle$ be an s.o.p. and $\alpha \in \mathscr{A}(R)$.

(1) Suppose $F\left(T_{1}, \ldots, T_{d}\right)$ is a relation on $\boldsymbol{x}$ and $1 \leq l \leq d$. Let $K=K(F)$ and $K^{\prime}=K\left(T_{l} F\right)$. For any choice of $b_{1}, \ldots, b_{K}$ there exist $b_{1}^{\prime}, \ldots, b_{K^{\prime}}^{\prime}$ such that

$$
\Phi_{\alpha, b_{1}^{\prime}, \ldots, b_{K^{\prime}}^{\prime}}\left(T_{l} F\left(T_{1}, \ldots, T_{d}\right)\right)=T_{l} \Phi_{\alpha, b_{1}, \ldots, b_{K}}\left(F\left(T_{1}, \ldots, T_{d}\right)\right) .
$$

(2) Suppose $F\left(T_{1}, \ldots, T_{d}\right)$ and $H\left(T_{1}, \ldots, T_{d}\right)$ are two relations on $\boldsymbol{x}$ of the same degree. Let $K^{\prime}=K(F), K^{\prime \prime}=K(H)$ and $K=K(F+H)$. There exist choices of $b_{1}, \ldots, b_{K}$, and $b_{1}^{\prime}, \ldots, b_{K^{\prime}}^{\prime}$ and $b_{1}^{\prime \prime}, \ldots, b_{K^{\prime \prime}}^{\prime \prime}$ such that

$$
\Phi_{\alpha, b_{1}, \ldots, b_{K}}(F+H)=\Phi_{\alpha, b_{1}^{\prime}, \ldots, b_{K^{\prime}}^{\prime}}(F)+\Phi_{\alpha, b_{1}^{\prime \prime}, \ldots, b_{K^{\prime \prime}}^{\prime \prime}}(H) .
$$

(3) Suppose $F\left(T_{1}, \ldots, T_{d}\right)$ is a relation on $\boldsymbol{x}$ and $P\left(T_{1}, \ldots, T_{d}\right)$ is any polynomial. Let $K=K(F)$ and $K^{\prime}=K(P F)$. There exist choices of $b_{1}, \ldots, b_{K^{\prime}}$ and $c_{1}, \ldots, c_{K}$ such that

$$
\begin{aligned}
\Phi_{\alpha, b_{1}, \ldots, b_{K^{\prime}}}\left(P\left(T_{1}, \ldots, T_{d}\right) F\left(T_{1}, \ldots, T_{d}\right)\right) & \\
& =P\left(T_{1}, \ldots, T_{d}\right) \Phi_{\alpha, c_{1}, \ldots, c_{K}}\left(F\left(T_{1}, \ldots, T_{d}\right)\right) .
\end{aligned}
$$


Proof. It is easy to see that (3) is a consequence of (1) and (2). We shall first prove (1). Suppose

$$
F\left(T_{1}, \ldots, T_{d}\right)=\sum_{n \leq n_{0}} r_{n} \boldsymbol{T}^{\boldsymbol{n}}
$$

with leading monomial $\boldsymbol{T}^{\boldsymbol{n}_{0}}$. Let $M=M(F)$ and $M^{\prime}=M\left(T_{l} F\right)$ be the presentation matrices associated to relations $F$ and $T_{l} F$, respectively, as obtained in Lemma 4.1. Suppose $C=\left[\begin{array}{lllllll}0 & \ldots & -x_{j} & \ldots & x_{i} & \ldots & 0\end{array}\right]^{\mathrm{T}}$ is a column of $M$ whose entries $-x_{j}$ and $x_{i}$ give the relation between monomials $\boldsymbol{x}^{n_{1}}$ and $\boldsymbol{x}^{\boldsymbol{n}_{2}}$ of $F\left(x_{1}, \ldots, x_{d}\right)$. Then, in $M^{\prime}$, there is a corresponding column $C^{\prime}=\left[\begin{array}{lllllll}0 & \ldots & -x_{j} & \ldots & x_{i} & \ldots & 0\end{array}\right]^{\mathrm{T}}$ (with more 0 's) whose $-x_{j}$ and $x_{i}$ entries give the relation between monomials $\boldsymbol{x}^{\boldsymbol{n}_{1}+\boldsymbol{e}_{l}}=x_{l} \boldsymbol{x}^{\boldsymbol{n}_{1}}$ and $\boldsymbol{x}^{\boldsymbol{n}_{2}+\boldsymbol{e}_{l}}=x_{l} \boldsymbol{x}^{\boldsymbol{n}_{2}}$ of $x_{l} F\left(x_{1}, \ldots, x_{d}\right)$. By reindexing, if necessary, we may assume that $C_{1}^{\prime}, \ldots, C_{K}^{\prime}$ are columns in $M^{\prime}$ corresponding to columns $C_{1}, \ldots, C_{K}$ of $M$. Now, in the presentation obtained from the relation $T_{l} F\left(T_{1}, \ldots, T_{d}\right)$ similar to (4-2), we may pick $b_{i}^{\prime}=b_{i}$ for $i=1, \ldots, K$ and $b_{i}^{\prime}=0$ for $i=K+1, \ldots, K^{\prime}$. (1) then follows from the construction of $\Phi$.

It remains to prove (2). Without loss of generality, we may assume that $K^{\prime} \geq K^{\prime \prime}$. If the leading terms of $F$ and $H$ do not cancel each other (which implies $K=K^{\prime}$ ), then (2) follows from the construction of functions $\Phi_{\alpha, b_{1}, \ldots, b_{K}}$ by taking the tuple

$$
\left(b_{1}, \ldots, b_{K}\right)=\left(b_{1}^{\prime}, \ldots, b_{K^{\prime}}^{\prime}\right)+(\underbrace{0, \ldots, 0}_{K^{\prime}-K^{\prime \prime}}, b_{1}^{\prime \prime}, \ldots, b_{K^{\prime \prime}}^{\prime \prime}) .
$$

Suppose now that $F\left(T_{1}, \ldots, T_{d}\right)=\sum_{\boldsymbol{m} \leq \boldsymbol{n}_{0}} r_{\boldsymbol{m}} \boldsymbol{T}^{\boldsymbol{m}}$ and $H=\sum_{\boldsymbol{m} \leq \boldsymbol{n}_{0}} r_{\boldsymbol{m}}^{\prime} \boldsymbol{T}^{\boldsymbol{m}}$, and $r_{n_{1}} \boldsymbol{T}^{\boldsymbol{n}_{1}}$ and $r_{\boldsymbol{n}_{1}}^{\prime} \boldsymbol{T}^{\boldsymbol{n}_{1}}$ are the highest terms in $F$ and $H$ that do not cancel (with $\boldsymbol{n}_{1}<\boldsymbol{n}_{0}$ ). In this case, $K<K^{\prime}=K^{\prime \prime}$ To prove (2), we only need to show that there are choices of $b_{1}, \ldots, b_{K}$ and $b_{1}^{\prime}, \ldots, b_{K^{\prime}}^{\prime}$ and $b_{1}^{\prime \prime}, \ldots, b_{K^{\prime \prime}}^{\prime \prime}$ such that the leading term of $\Phi_{\alpha, b_{1}^{\prime}, \ldots, b_{K^{\prime}}^{\prime}}(F)+\Phi_{\alpha, b_{1}^{\prime \prime}, \ldots, b_{K^{\prime \prime}}^{\prime \prime}}(H)$ is the same as that of $\Phi_{\alpha, b_{1}, \ldots, b_{K}}(F+H)$, which is $\left(r_{\boldsymbol{n}_{1}}+r_{\boldsymbol{n}_{1}}^{\prime}\right) \boldsymbol{T}^{\boldsymbol{n}_{1}}$.

Let $C_{1}, \ldots, C_{K^{\prime}}\left(K^{\prime}=K^{\prime \prime}\right)$ be the columns of the matrix of $\partial_{2}$ 's corresponding to $F$ and $H$ as in Lemma 4.1. Suppose $b_{1}^{\prime}, \ldots, b_{K^{\prime}}^{\prime}$ and $b_{1}^{\prime \prime}, \ldots, b_{K^{\prime \prime}}^{\prime \prime}$ are the coefficients in equalities of the form (4-2) corresponding to $F$ and $H$. We have

$$
\left(\begin{array}{c}
0 \\
\vdots \\
\alpha\left(r_{\boldsymbol{n}_{0}}+r_{\boldsymbol{n}_{0}}^{\prime}\right) \\
\vdots \\
\alpha\left(r_{\boldsymbol{n}_{1}}+r_{\boldsymbol{n}_{1}}^{\prime}\right) \\
\vdots
\end{array}\right)=\left(b_{1}^{\prime}+b_{1}^{\prime \prime}\right) C_{1}+\cdots+\left(b_{K^{\prime}}^{\prime}+b_{K^{\prime \prime}}^{\prime \prime}\right) C_{K^{\prime}}
$$

Suppose in (4-4), $C_{1}, \ldots, C_{L}$ are columns that have at least a nonzero entry higher than $\alpha\left(r_{n_{1}}+r_{n_{1}}^{\prime}\right)$. Then, $b_{1}^{\prime}+b_{1}^{\prime \prime}=\cdots=b_{L}^{\prime}+b_{L}^{\prime \prime}=0$. It can be easily seen that 
$K=K^{\prime}-L$. We can now pick $b_{1}=b_{L+1}^{\prime}+b_{L+1}^{\prime \prime}, \ldots, b_{K}=b_{K^{\prime}}^{\prime}+b_{K^{\prime \prime}}^{\prime \prime}$ and the required equality follows from (4-4). The lemma is proved.

Theorem 4.3 (compare [Lai 1995, Theorem 3.1]). Let $(R, \mathfrak{m}, k)$ be a local ring. Assume that $\boldsymbol{x}=\left\langle x_{1}, \ldots, x_{d}\right\rangle$ and $\boldsymbol{y}=\left\langle y_{1}, x_{2}, \ldots, x_{d}\right\rangle$ are both s.o.p.'s such that $\alpha=y_{1}-x_{1} \in \mathscr{A}(R)$. Then

$$
\operatorname{rt}\left(x_{1}, \ldots, x_{d}\right)=\operatorname{rt}\left(y_{1}, x_{2}, \ldots, x_{d}\right) .
$$

Proof. It is enough to show that $\operatorname{rt}\left(x_{1}, \ldots, x_{d}\right) \leq \operatorname{rt}\left(y_{1}, x_{2}, \ldots, x_{d}\right)$ (since we then can apply the inequality for $-\alpha)$. Suppose that $r=\operatorname{rt}\left(y_{1}, x_{2}, \ldots, x_{d}\right)$. Let $F\left(T_{1}, \ldots, T_{d}\right)$ be a relation on $x_{1}, \ldots, x_{d}$ of degree $N>r$. Let $K=K(F)$ and let $\tilde{F}_{b_{1}, \ldots, b_{K}}\left(T_{1}, \ldots, T_{d}\right)=\Phi_{\alpha, b_{1}, \ldots, b_{K}}\left(F\left(T_{1}, \ldots, T_{d}\right)\right)$ for some choice of $b_{1}, \ldots, b_{K}$. Then, as shown before, $\tilde{F}_{b_{1}, \ldots, b_{K}}$ gives a relation on $\boldsymbol{y}=\left(y_{1}, x_{2}, \ldots, x_{d}\right)$. Since $\tilde{F}_{b_{1}, \ldots, b_{K}}$ has degree $N>r$, it can be written as

$$
\tilde{F}_{b_{1}, \ldots, b_{K}}\left(T_{1}, \ldots, T_{d}\right)=\sum_{i=1}^{m} P_{i}\left(T_{1}, \ldots, T_{d}\right) H_{i}\left(T_{1}, \ldots, T_{d}\right),
$$

where the $P_{i}$ 's are polynomial in $T_{1}, \ldots, T_{d}$ and the $H_{i}$ 's provide relations on $\boldsymbol{y}=\left(y_{1}, x_{2}, \ldots, x_{d}\right)$ with $\operatorname{deg} H_{i} \leq r$ for all $i$. For $i=1, \ldots, m$, let $K_{i}=K\left(H_{i}\right)$. It follows from Lemma 4.2 that there exist choices of $c_{1}, \ldots, c_{K}$ and $b_{i 1}, \ldots, b_{i K_{i}}$ such that

$$
\Phi_{-\alpha, c_{1}, \ldots, c_{K}}\left(\tilde{F}_{b_{1}, \ldots, b_{K}}\right)=\sum_{i=1}^{m} P_{i}\left(T_{1}, \ldots, T_{d}\right) \Phi_{-\alpha, b_{i 1}, \ldots, b_{i K_{i}}}\left(H_{i}\left(T_{1}, \ldots, T_{d}\right)\right) .
$$

By the definition, $\Phi_{-\alpha, b_{i 1}, \ldots, b_{i K_{i}}}\left(H_{i}\left(T_{1}, \ldots, T_{d}\right)\right)$ gives a relation on $x_{1}, \ldots, x_{d}$ for each $i$, and

$$
\operatorname{deg} \Phi_{-\alpha, b_{i 1}, \ldots, b_{i K_{i}}}\left(H_{i}\left(T_{1}, \ldots, T_{d}\right)\right) \leq r .
$$

Moreover, since $\tilde{F}_{b_{1}, \ldots, b_{K}}$ and $F$ have the same leading term, $\Phi_{-\alpha, c_{1}, \ldots, c_{K}}\left(\tilde{F}_{b_{1}, \ldots, b_{K}}\right)$ and $F$ also have the same leading term. Thus, we can write

$$
F\left(T_{1}, \ldots, T_{d}\right)=\sum_{i=1}^{m} P_{i}\left(T_{1}, \ldots, T_{d}\right) \Phi_{-\alpha, b_{i 1}, \ldots, b_{i K_{i}}}\left(H_{i}\left(T_{1}, \ldots, T_{d}\right)\right)+F^{\prime}\left(T_{1}, \ldots, T_{d}\right),
$$

where $F^{\prime}\left(T_{1}, \ldots, T_{d}\right)$ gives a relation on $x_{1}, \ldots, x_{d}$ and has a smaller leading term than that of $F\left(T_{1}, \ldots, T_{d}\right)$. Repeating this process, we eventually will get to the situation when $F^{\prime}\left(T_{1}, \ldots, T_{d}\right)=0$, or equivalently, when $F\left(T_{1}, \ldots, T_{d}\right)$ is a combination of relations on $x_{1}, \ldots, x_{d}$ with degrees at most $r$. Hence, $\operatorname{rt}\left(x_{1}, \ldots, x_{d}\right) \leq$ $r=\operatorname{rt}\left(y_{1}, x_{2}, \ldots, x_{d}\right)$. The theorem is proved.

Now recall from Definition 3.8 the notion of a superficial sequence. 
Lemma 4.4. Let $\boldsymbol{x}=\left\langle x_{1}, \ldots, x_{d}\right\rangle$ be an s.o.p. such that $x_{d}$ is superficial for (x). Suppose $\boldsymbol{y}=\left\langle x_{1}, \ldots, x_{d-1}, y_{d}\right\rangle$ is an s.o.p. such that $\alpha=y_{d}-x_{d} \in \mathscr{A}(R)$. Then $y_{d}$ is superficial for $(\boldsymbol{y})$.

Proof. Suppose $c \in \mathbb{N}$ is an integer such that $\left[(\boldsymbol{x})^{n}: x_{d}\right] \cap(\boldsymbol{x})^{c}=(\boldsymbol{x})^{n-1}$ for all $n>c$. Also, let $k \in \mathbb{N}$ be an integer given by the Artin-Rees lemma for the modules $x_{d} R \subseteq R$ and the ideal $\left(x_{1}, \ldots, x_{d-1}\right)$, i.e., for $m \geq k$ we have

$$
\left(x_{1}, \ldots, x_{d-1}\right)^{m} \cap\left(x_{d}\right) \subseteq x_{d}\left(x_{1}, \ldots, x_{d-1}\right)^{m-1} .
$$

We first observe that $\left(0: x_{d}\right) \subseteq H_{\mathfrak{m}}^{0}(R)$ since if $x_{d} u=0$ we have $x_{d}(\boldsymbol{x})^{c} u=0 \subseteq(\boldsymbol{x})^{n}$ for all $n>c$. By superficiality, $(\boldsymbol{x})^{c} u \in(\boldsymbol{x})^{n-1}$ for all $n \gg 0$, so by the Krull intersection theorem, $(\boldsymbol{x})^{c} u=0$. Since $(\boldsymbol{x})$ is $\mathfrak{m}$-primary, $u \in H_{\mathfrak{m}}^{0}(R)$. We let $t \in \mathbb{N}$ be such that $\mathfrak{m}^{t} \cap H_{\mathfrak{m}}^{0}(R)=0$.

We will now show that if $s \in(\boldsymbol{y})^{c+k+t}$ and $y_{d} s \in(\boldsymbol{y})^{m}$ for $m>c+k+t$ then $s \in(\boldsymbol{y})^{m-1}$. Since we then have $y_{d}\left(s-s^{\prime}\right) \in\left(x_{1}, \ldots, x_{d-1}\right)^{m}$ where $s^{\prime} \in(\boldsymbol{y})^{m-1}$, it suffices to assume that $s \in\left(x_{1}, \ldots, x_{d-1}\right)^{m}: y_{d}$. Since $\alpha$ is a homology multiplier, we then have $\alpha s \in\left(x_{1}, \ldots, x_{d-1}\right)^{m}$. Thus,

$$
s x_{d}=s y_{d}-\alpha s \in\left(x_{1}, \ldots, x_{d-1}\right)^{m} .
$$

From the Artin-Rees lemma we see that

$$
s x_{d} \in\left(x_{1}, \ldots, x_{d-1}\right)^{m} \cap\left(x_{d}\right) \subseteq x_{d}\left(x_{1}, \ldots, x_{d-1}\right)^{m-k} ;
$$

hence $s \in\left[\left(x_{1}, \ldots, x_{d-1}\right)^{m-k}+\left(0: x_{d}\right)\right] \cap(y)^{t}$. If we write $s=s_{1}+s_{2}$ where $s_{2} \in\left(0: x_{d}\right)$, then $s_{2} \in\left(0: x_{d}\right) \cap \mathfrak{m}^{t} \subseteq H_{\mathfrak{m}}^{0}(R) \cap \mathfrak{m}^{t}=0$ (since $\left.m-k \geq t\right)$. Thus $s \in(\boldsymbol{x})^{c}$, and since $x_{d}$ is superficial for $(\boldsymbol{x})$, we must have $s \in\left(x_{1}, \ldots, x_{d}\right)^{m-1}$. Set

$$
s=\sum_{|n|=m-1} a_{n} x^{n}
$$

We can now write (4-5) as

$$
\sum_{|n|=m-1} a_{n} x^{n} x_{d}-\sum_{|n|=m} d_{n} x^{n}=0
$$

where $Q\left(x_{1}, \ldots, x_{d-1}\right)=\sum_{|\boldsymbol{n}|=m} d_{n} x^{n} \in\left(x_{1}, \ldots, x_{d-1}\right)^{m}$. Let $P\left(x_{1}, \ldots, x_{d}\right)=$ $\sum_{|\boldsymbol{n}|=m-1} a_{\boldsymbol{n}} \boldsymbol{x}^{\boldsymbol{n}}$. Then, (4-6) gives a relation on $x_{1}, \ldots, x_{d}$ of degree $m$, namely

$$
H\left(T_{1}, \ldots, T_{d}\right)=T_{d} P\left(T_{1}, \ldots, T_{d}\right)-Q\left(T_{1}, \ldots, T_{d-1}\right) .
$$

Let $r_{\boldsymbol{m}_{0}} \boldsymbol{T}^{\boldsymbol{m}_{0}}$ (where $\left|\boldsymbol{m}_{0}\right|=m$ ) be the leading term of $H\left(T_{1}, \ldots, T_{d}\right)$. It follows from Lemma 4.1 that

$$
H\left(x_{1}, \ldots, x_{d}\right)-H\left(x_{1}, \ldots, x_{d-1}, y_{d}\right) \in\left(\boldsymbol{y}^{\boldsymbol{m}} \mid \boldsymbol{m}<\boldsymbol{m}_{0}\right) .
$$


Moreover,

$H\left(x_{1}, \ldots, x_{d}\right)-H\left(x_{1}, \ldots, x_{d-1}, y_{d}\right)=x_{d} P\left(x_{1}, \ldots, x_{d}\right)-y_{d} P\left(x_{1}, \ldots, x_{d-1}, y_{d}\right)$.

Thus

$$
x_{d} P\left(x_{1}, \ldots, x_{d}\right)-y_{d} P\left(x_{1}, \ldots, x_{d-1}, y_{d}\right) \in\left(\boldsymbol{y}^{\boldsymbol{m}} \mid \boldsymbol{m}<\boldsymbol{m}_{0}\right) .
$$

Since the calculation done in Lemma 4.1 is formal on the coefficients of the relation $F\left(T_{1}, \ldots, T_{d}\right)$, in our situation it is formal on the coefficients of $T_{d} P\left(T_{1}, \ldots, T_{d}\right)$, which are exactly the same as those of $P\left(T_{1}, \ldots, T_{d}\right)$. Therefore, the same calculation as in (4-7) would hold for $P\left(x_{1}, \ldots, x_{d}\right)$. Hence, for $\boldsymbol{m}_{0}^{\prime}=\boldsymbol{m}_{0}-(0, \ldots, 0,1)$, we have

$$
P\left(x_{1}, \ldots, x_{d}\right)-P\left(x_{1}, \ldots, x_{d-1}, y_{d}\right) \in\left(\boldsymbol{y}^{\boldsymbol{m}} \mid \boldsymbol{m}<\boldsymbol{m}_{0}^{\prime}\right) .
$$

It follows from this that

$$
s=P\left(x_{1}, \ldots, x_{d}\right) \in\left(x_{1}, \ldots, x_{d-1}, y_{d}\right)^{m-1} .
$$

Lemma 4.5. Let $\boldsymbol{x}$ and $\boldsymbol{y}$ be as in Lemma 4.4 in a local ring $(R, \mathfrak{m})$ with infinite residue field. There exist $y_{1}^{\prime}, \ldots, y_{d-1}^{\prime}$ such that $\left(y_{1}^{\prime}, \ldots, y_{d-1}^{\prime}, y_{d}\right)=(\boldsymbol{y})$, and $y_{d}, y_{d-1}^{\prime}, \ldots, y_{1}^{\prime}$ is a superficial sequence.

Proof. Take $y_{1}^{\prime}, \ldots, y_{d-1}^{\prime}$ to be general linear combinations of $x_{1}, \ldots, x_{d-1}$.

\section{Ramsey numbers}

In this section, we provide a "Ramsey number" combinatorial lemma which will be used to establish uniform bounds on relation type of parameter ideals in the next two sections.

For a set $S$, and a positive integer $l$, we denote by $[S]^{l}$ the set of all subsets of $l$ elements of $S$. We shall use the following infinite version of Ramsey's theorem [1929] (see also [Adhikari 2002, Theorem 3.4]).

Lemma 5.1. Let $n$ and $l$ be two given positive integers. Let $T=\left\{x_{1}, x_{2}, \ldots\right\}$ be an infinite countable set. Then for any way of coloring $[T]^{l}$ using $n$ colors, there is an infinite subset $U$ of $T$ with all its subsets of l elements having the same color.

For any $d$-uple $A=\left(a_{1}, \ldots, a_{d}\right)$ of nonnegative integers, we denote by $|A|$ the sum $\sum_{j=1}^{d} a_{j}$. For two $d$-uples of nonnegative integers $A=\left(a_{1}, \ldots, a_{d}\right)$ and $B=\left(b_{1}, \ldots, b_{d}\right)$, we write $A \preceq B$ if and only if $a_{j} \leq b_{j}$ for all $j=1, \ldots, d$. A chain of length $l$ is a sequence of $d$-tuples of nonnegative integers $A_{1} \preceq A_{2} \preceq \cdots \preceq A_{l}$. For a $d$-uple $A=\left(a_{1}, \ldots, a_{d}\right)$ and a number $t \in\{1, \ldots, d\}$, we use $A(t)$ to denote the $t$-th entry $a_{t}$ of $A$. 
Lemma 5.2. Suppose $d$ and $l$ are given positive integers, and $k$ is a nonnegative integer. Then, there exists a positive number $M=M(d, k, l)$ such that for any sequence of $d$-uples of nonnegative integers $\mathscr{A}=\left\langle A_{1}, A_{2}, \ldots, A_{M}\right\rangle$, in which $\left|A_{i}\right|=k+i$, we can always find a chain $A_{i_{1}} \preceq A_{i_{2}} \preceq \cdots \preceq A_{i_{l}}$ of length $l$ with $1 \leq i_{1}<i_{2}<\cdots<i_{l} \leq M$.

Proof. By contradiction, suppose the assertion is not true. That is, for any $M \geq k$, there is a sequence $\mathscr{A}_{M}=\left\langle A_{M, 1}, A_{M, 2}, \ldots, A_{M, M}\right\rangle$ of $d$-uples of nonnegative integers such that $\left|A_{M, i}\right|=k+i$ for $i=1, \ldots, M$, which has no subchain of length $l$.

We shall first inductively construct an infinite sequence of infinite subsets $M_{1} \supseteq$ $M_{2} \supseteq \cdots \supseteq M_{n} \supseteq \cdots$ of $\mathbb{N}$ as follows. Since $\left|A_{M, 1}\right|=k+1$ for all $M \geq k$, there must be an infinite subset $\mathcal{M}_{1}$ of $\mathbb{N}$ such that for any $M, N \in \mathcal{M}_{1}$, we have $A_{M, 1}=A_{N, 1}$. Suppose $M_{1}, \ldots, M_{i}(i \geq 1)$ have been constructed. Since for each $M \in \mathcal{M}_{i},\left|A_{M, i+1}\right|=k+i+1$, there must be an infinite subset $M_{i+1}$ of $M_{i}$ such that for any $M, N \in M_{i+1}$, we have $A_{M, i+1}=A_{N, i+1}$.

Let $\mathscr{A}=\left\langle A_{1}, A_{2}, \ldots\right\rangle$ be the sequence defined by letting $A_{i}=A_{M, i}$, where $M$ is an arbitrary element of $\mathcal{M}_{i}$. Clearly, by definition, $\left|A_{i}\right|=k+i$.

Claim 5.3. The sequence $\mathscr{A}=\left\langle A_{1}, A_{2}, \ldots\right\rangle$ does not have a subchain of length $l$.

Proof of Claim. Indeed, if $B_{1} \preceq B_{2} \preceq \cdots \preceq B_{l}$ is a subchain of $\mathscr{A}$, and $B_{l}=A_{s}$, then for any $M \in \mathcal{M}_{s}$, the sequence $\mathscr{A}_{M}=\left\langle A_{M, 1}, A_{M, 2}, \ldots\right\rangle$ contains the subchain $B_{1} \preceq B_{2} \preceq \cdots \preceq B_{l}$ of length $l$. This is a contradiction.

Now consider a coloring of $[A]^{2}$ using $(d+1)$ colors as follows. Suppose $i<j$. If $A_{i} \preceq A_{j}$, then we color $\left\{A_{i}, A_{j}\right\}$ by 0 . Otherwise, there must be an integer $t \in\{1, \ldots, n\}$ such that $A_{i}(s) \leq A_{j}(s)$ for any $s<t$ and $A_{i}(t)>A_{j}(t)$, and we color $\left\{A_{i}, A_{j}\right\}$ by $t$. Clearly, this is a valid $(d+1)$-coloring of $[\mathscr{A}]^{2}$. By Ramsey's theorem (Lemma 5.1), there exists an infinite subset $U=\left\{U_{1}, U_{2}, \ldots\right\}$ of $\mathscr{A}$ such that $[u]^{2}$ has one color. Suppose $[u]^{2}$ is colored by $c$. If $c>0$, then we obtain an infinite sequence of nonnegative integers $U_{1}(c)>U_{2}(c)>\ldots$, which is impossible. Thus, $c=0$, and so $U_{1} \preceq U_{2} \preceq \ldots$ is a chain of infinite length in $\mathscr{A}$. But this is impossible by the Claim.

Hence, we always get a contradiction. The lemma is proved.

\section{Relation type in generalized Cohen-Macaulay rings}

The goal of this section will be to give a new argument that rings of finite local cohomology have uniformly bounded relation type. Throughout this section, $(R, \mathfrak{m}, k)$ will denote a local ring $R$ with maximal ideal $\mathfrak{m}$ and residue field $k$.

We recall that a Noetherian local ring $R$ of dimension $d$ is said to have finite local cohomology (f.l.c.) if $H_{\mathfrak{m}}^{i}(R)$ is finitely generated for $i=0, \ldots, d-1$ (and hence 
is of finitely length). Rings with finite local cohomology are called generalized Cohen-Macaulay. We observe that $R$ has f.l.c. if and only if $\hat{R}$ has f.l.c. if and only if $\operatorname{dim} \hat{R} / p=\operatorname{dim} \hat{R}$ for every minimal prime $p$ of $\hat{R}$, and $\hat{R}_{p}$ is Cohen-Macaulay for all $p \neq \hat{\mathfrak{m}}$.

Notice that if a Noetherian local ring $R$ has f.l.c. then $\mathscr{A}(R)$ is $\mathfrak{m}$-primary.

We shall start with a result in two dimensions, through which the argument in the general situation becomes more transparent. (The bound $l_{1}+l_{0}$ in this special case improves the bound $2 l_{1}+l_{0}$ of [Wang 1997a, Theorem 4.1].)

Theorem 6.1. Let $(R, \mathfrak{m}, k)$ be an equidimensional local ring of dimension 2 such that $\lambda\left(H_{\mathfrak{m}}^{0}(R)\right)=l_{0}<\infty$ and $\lambda\left(H_{\mathfrak{m}}^{1}(R)\right)=l_{1}<\infty$. Then $R$ has a uniform bound $l_{1}+l_{0}$ on relation type of parameter ideals.

Proof. Let $I=(x, y)$ be a parameter ideal of $R$. By [Wang 1997a, Lemma 2.2] we can assume that $H_{\mathfrak{m}}^{0}(R)=0$ and show that $\operatorname{rt}(I) \leq l_{1}$. Suppose

$$
F\left(T_{1}, T_{2}\right)=r_{N} T_{1}^{N}+r_{N-1} T_{1}^{N-1} T_{2}+\cdots+r_{0} T_{2}^{N},
$$

with $N>l_{1}$, provides a relation on $(x, y)$. That is,

$$
r_{N} x^{N}+r_{N-1} x^{N-1} y+\cdots+r_{0} y^{N}=0 .
$$

We may assume that $r_{N} \neq 0$, otherwise we can factor out a power of $T_{2}$.

Let $\gamma \in \mathscr{A}(R)$ be a homology multiplier which is part of an s.o.p. (in particular, $\gamma$ is not a zero-divisor). Let $l=\lambda\left(H_{\mathfrak{m}}^{0}(R / \gamma R)\right)$. By [Wang 1997a, Lemma 3.7] we have that $l \leq l_{1}$. It follows from (6-1) that $r_{N} \in y: x^{N}$. Thus, since $\gamma$ is a homology multiplier, we have

$$
\gamma r_{N} \in(y) .
$$

Similarly, (6-1) implies that $r_{N} x+r_{N-1} y \in y^{2}: x^{N-1}$, and so

$$
\gamma\left(r_{N} x+r_{N-1} y\right) \in\left(y^{2}\right) .
$$

Proceeding in this way, we obtain a sequence of relations as follows.

$$
\begin{aligned}
& \gamma r_{N}=s_{1} y, \quad \gamma\left(r_{N} x+r_{N-1} y\right)=s_{2} y^{2}, \quad \ldots, \\
& \gamma\left(r_{N} x^{N-1}+r_{N-1} x^{N-2} y+\cdots+r_{1} y^{N-1}\right)=s_{N} y^{N},
\end{aligned}
$$

where $s_{1}, \ldots, s_{N} \in R$.

For every $1 \leq j \leq N$, from (6-2) we have $s_{j} \in\left(\gamma: y^{j}\right) \subseteq H_{\mathfrak{m}}^{0}(R / \gamma R)$. Since $N>l_{1}$, there exists $p \leq l_{1}$ such that

Write

$$
s_{p+1} \in\left(\gamma, s_{1}, \ldots, s_{p}\right) .
$$

$$
s_{p+1}=a \gamma+b_{1} s_{1}+\cdots+b_{p} s_{p} .
$$


Substituting this into the relation $\gamma\left(r_{N} x^{p}+r_{N-1} x^{p-1} y+\cdots+r_{N-p} y^{p}\right)=s_{p+1} y^{p+1}$ in (6-2), we obtain

$$
\begin{aligned}
\gamma\left(r_{N} x^{p}+r_{N-1} x^{p-1} y+\cdots+r_{N-p} y^{p}\right) & =\left(a \gamma+b_{1} s_{1}+\cdots+b_{p} s_{p}\right) y^{p+1} \\
& =a \gamma y^{p+1}+b_{1} s_{1} y^{p+1}+\cdots+b_{p} s_{p} y^{p+1},
\end{aligned}
$$

that is,

$$
\begin{aligned}
\gamma\left(r_{N} x^{p}+r_{N-1} x^{p-1} y+\cdots+\right. & \left.\left(r_{N-p}-a y\right) y^{p}\right) \\
& =\left(b_{1} y^{p}\right) s_{1} y+\left(b_{2} y^{p-1}\right) s_{2} y^{2}+\cdots+\left(b_{p} y\right) s_{p} y^{p} .
\end{aligned}
$$

Using (6-2), replace each term $\left(b_{j+1} y^{p-j}\right) s_{j+1} y_{j+1}$ on the right-hand side with $\gamma\left(\left(r_{N} b_{j+1} y^{p-j}\right) x^{j}+\cdots+\left(r_{N-j} b_{j+1} y^{p-j}\right) y^{j}\right)$. Moving everything to the lefthand side and observing that $\left(r_{N} b_{j+1} y^{p-j}\right) x^{j}=\left(r_{N} b_{j+1}\right) x^{j} y^{p-j}<r_{N} x^{p}$, for each $j=1, \ldots, p-1$, we obtain, upon factoring out $\gamma$,

$$
\gamma\left(r_{N} x^{p}+\text { smaller terms }\right)=0 .
$$

But $\gamma$ is not a zero-divisor, so

$$
r_{N} x^{p}+\text { smaller terms }=0 .
$$

We thus get a new relation on $(x, y)$,

$$
G\left(T_{1}, T_{2}\right)=r_{N} T_{1}^{p}+\text { smaller terms }
$$

which has the same leading coefficient as $F\left(T_{1}, T_{2}\right)$ but is of lower degree.

Now, write $F=T_{1}^{N-p} G\left(T_{1}, T_{2}\right)+T_{2} H\left(T_{1}, T_{2}\right)$. Then, clearly $H\left(T_{1}, T_{2}\right)$ also provides a relation on $(x, y)$ and is of smaller degree than $F\left(T_{1}, T_{2}\right)$. That is, $F\left(T_{1}, T_{2}\right)$ can be written as a combination of relations of lower degrees. This proves our result.

To prove the result for generalized CM rings of any dimension, we shall need the following lemma of Schenzel (see [Wang 1997a, Theorem 3.2]).

Lemma 6.2. Let $(R, \mathfrak{m}, k)$ be a d-dimensional Noetherian ring having finite local cohomology. Let $\left(x_{1}, \ldots, x_{s}\right)$ be part of an s.o.p. in $R$. Then,

$$
\lambda\left(\frac{\left(x_{2}, \ldots, x_{s}\right): x_{1}}{\left(x_{2}, \ldots, x_{s}\right)}\right) \leq \sum_{i=0}^{s-1}\left(\begin{array}{c}
s-1 \\
i
\end{array}\right) \lambda\left(H_{\mathfrak{m}}^{i}(R)\right) .
$$

Remark 6.3. Under the assumptions of Lemma 6.2, we have

$$
H_{\mathfrak{m}}^{0}\left(R /\left(x_{2}, \ldots, x_{s}\right)\right)=\frac{\left(x_{2}, \ldots, x_{s}\right): x_{1}^{\infty}}{\left(x_{2}, \ldots, x_{s}\right)}=\frac{\left(x_{2}, \ldots, x_{s}\right): x_{1}^{n}}{\left(x_{2}, \ldots, x_{s}\right)},
$$


for some positive integer $n$. Since if $\left(x_{1}, \ldots, x_{s}\right)$ is part of an s.o.p. then so is $\left(x_{1}^{n}, x_{2}, \ldots, x_{s}\right)$, Lemma 6.2 now gives

$$
\lambda\left(H_{\mathfrak{m}}^{0}\left(R /\left(x_{2}, \ldots, x_{s}\right)\right)\right) \leq \sum_{i=0}^{s-1}\left(\begin{array}{c}
s-1 \\
i
\end{array}\right) \lambda\left(H_{\mathfrak{m}}^{i}(R)\right) .
$$

Our result for generalized CM rings of any dimension is stated as follows.

Theorem 6.4. Let $(R, \mathfrak{m}, k)$ be a local ring of dimension d such that $\lambda\left(H_{\mathfrak{m}}^{i}(R)\right)<$ $\infty$ for $0 \leq i \leq d-1$. Then $R$ has a uniform bound on relation type of parameter ideals.

Proof. By [Wang 1997b, Lemma 4.1], since our hypotheses pass to the completion, we may assume that $R$ is complete. By [Wang 1997a, Lemma 2.2], we may also assume that $H_{\mathfrak{m}}^{0}(R)=0$. Next, by passing to a faithful extension of $R$ if necessary, we may assume that $k$ is infinite; see the proof of [Wang 1997a, Theorem 4.4].

Let $I=\left(x_{1}, \ldots, x_{d}\right)$ be a parameter ideal in $R$. We can pick $x_{d}, x_{d-1}, \ldots, x_{1}$ to form a superficial sequence and $x_{d}$ not to be a zero-divisor. We will use graded reverse lex monomial ordering with $x_{1}>x_{2}>\cdots>x_{d}$ and $T_{1}>T_{2}>\cdots>T_{d}$. For $q=2, \ldots, d$, let

$$
L_{q}=\left(\sum_{i=0}^{d-q+1}\left(\begin{array}{c}
d-q+1 \\
i
\end{array}\right) \lambda\left(H_{\mathfrak{m}}^{i}(R)\right)\right)+1 .
$$

Set $L=\left(L_{2}-1\right)+\cdots+\left(L_{d}-1\right)+1$. Let $M(d, k, l)$ denote the Ramsey number determined as in Lemma 5.2, and set $K_{1}=M(d, 1, L)=N_{1}$. We recursively construct three sequences of finite numbers: for $i \geq 2$, let

$$
M_{i}=\sum_{l=0}^{K_{i-1}}\left(\begin{array}{c}
l+i-2 \\
i-2
\end{array}\right)
$$

be the number of all $(i-1)$-tuples of nonnegative integers whose sum is at most $K_{i-1}$; then let $N_{i}=M\left(d, 2 K_{i-1}, M_{i}(L-1)+1\right)$ and $K_{i}=2 K_{i-1}+N_{i}$.

To get the conclusion, it suffices to prove that any relation on $x_{1}, \ldots, x_{d}$ of degree greater than $K_{d-1}$ can be written as a combination of relations of smaller degrees. Consider an arbitrary relation of degree $N>K_{d-1}$ in $x_{1}, \ldots, x_{d}$ :

That is,

$$
F=F\left(T_{1}, \ldots, T_{d}\right)=\sum_{|\boldsymbol{n}|=N} r_{\boldsymbol{n}} \boldsymbol{T}^{\boldsymbol{n}} .
$$

$$
F\left(x_{1}, \ldots, x_{d}\right)=\sum_{|\boldsymbol{n}|=N} r_{n} x^{n}=0
$$


We can assume that $T_{d}$ does not divide the leading term of $F$, since otherwise we can factor out $T_{d}$ and get a relation of smaller degree (since $x_{d}$ is not a zero-divisor). Let $\operatorname{ord}_{i}\left(\boldsymbol{T}^{\boldsymbol{n}}\right)$ be the $i$-th component of $\boldsymbol{n}$, and let $\operatorname{ord}_{<j}\left(\boldsymbol{T}^{\boldsymbol{n}}\right)=\sum_{i<j} \operatorname{ord}_{i}\left(\boldsymbol{T}^{\boldsymbol{n}}\right)$. We shall show that for any $1 \leq j \leq d-1$, if $F$ contains a term $r_{\boldsymbol{m}} \boldsymbol{T}^{\boldsymbol{m}}$ (say $\boldsymbol{m}=$ $\left.\left(m_{1}, \ldots, m_{d}\right)\right)$ with $\operatorname{ord}_{<j+1}\left(\boldsymbol{T}^{\boldsymbol{m}}\right)>K_{j}$ then we can write

$$
F\left(T_{1}, \ldots, T_{d}\right)=H\left(T_{1}, \ldots, T_{d}\right) G\left(T_{1}, \ldots, T_{d}\right)+F^{\prime}\left(T_{1}, \ldots, T_{d}\right),
$$

where $H$ is a monomial divisible by $\prod_{i>j} T_{i}^{m_{i}}, G$ and $F^{\prime}$ both provide relations in $x_{1}, \ldots, x_{d}$, the leading term $r_{k} T^{k}$ of $G$ satisfies $r_{k} T^{k} \mid r_{m} T^{m}$, and further $\operatorname{ord}_{<j+1}\left(\boldsymbol{T}^{k}\right) \leq K_{j}$ and $\operatorname{ord}_{j+1}\left(\boldsymbol{T}^{k}\right)=\cdots=\operatorname{ord}_{d}\left(\boldsymbol{T}^{\boldsymbol{k}}\right)=0$ (in particular, the degree of $G$ is bounded by $\left.K_{j}\right)$, and all terms $r_{n_{1}} \boldsymbol{T}^{\boldsymbol{n}_{1}}$ of $F^{\prime}$ with $\operatorname{ord}_{<j+1}\left(\boldsymbol{T}^{\boldsymbol{n}_{1}}\right)>K_{j}$ are smaller than $r_{m} T^{m}$. By taking $j=d-1$, and successively eliminating terms $r_{n} T^{n}$ of $F$ with ord $\operatorname{od}_{<d}\left(\boldsymbol{T}^{n}\right)>K_{d-1}$, we then prove our theorem.

Suppose our assertion is not true. Let $j$ be the smallest index for which our assertion fails. Let $F$ be a relation such that our assertion fails for this value of $j$. In particular, $F$ contains a term $r_{\boldsymbol{n}} \boldsymbol{T}^{\boldsymbol{n}}$ such that ord ${ }_{<j+1}\left(\boldsymbol{T}^{\boldsymbol{n}}\right)>K_{j}$. Let $r_{\boldsymbol{n}_{0}} \boldsymbol{T}^{\boldsymbol{n}_{0}}$ be the largest term of $F$ for which ord $\operatorname{coj}_{<j+1}\left(\boldsymbol{T}^{\boldsymbol{n}_{0}}\right)>K_{j}$. Among all such relations $F$ 's for which our assertion fails for $j$, we shall pick $F$ such that $r_{n_{0}} \boldsymbol{T}^{\boldsymbol{n}_{0}}$ is smallest possible. Let $\boldsymbol{n}_{0}=\left(n_{01}, \ldots, n_{0 d}\right)$. For simplicity, we write $r$ for $r_{\boldsymbol{n}_{0}}$. We shall derive a contradiction.

From the choice of $j$, we may assume that

$$
K=\sum_{i=1}^{j-1} n_{0 i} \leq K_{j-1}
$$

We first observe that

$$
n_{0 j}>K_{j}-K_{j-1}=K_{j-1}+N_{j} .
$$

Let $\boldsymbol{J}$ denote the set of all monomials in $T_{1}, \ldots, T_{d}$ that appear in the expression of $F\left(T_{1}, \ldots, T_{d}\right)$. Let

$$
P\left(T_{1}, \ldots, T_{d}\right):=\prod_{i>j} T_{i}^{n_{0 i}}\left(r \frac{\boldsymbol{T}^{\boldsymbol{n}_{0}}}{\prod_{i>j} T_{i}^{n_{0 i}}}+\sum_{\substack{\boldsymbol{n} \in \boldsymbol{J}, \boldsymbol{n} \neq \boldsymbol{n}_{0}, \prod_{i>j} T_{i}^{n_{0 i}} \mid \boldsymbol{T}^{\boldsymbol{n}}}} r_{\boldsymbol{n}} \frac{\boldsymbol{T}^{\boldsymbol{n}}}{\prod_{i>j} T_{i}^{n_{0 i}}}\right)
$$

be the sum of all terms of $F$ divisible by $\prod_{i>j} T_{i}^{n_{0 i}}$. We first observe that if $\boldsymbol{n}>\boldsymbol{n}_{0}$, then from the choice of $r_{n_{0}} T^{n_{0}}$, we must have ord $\operatorname{orj+1}_{<j}\left(T^{n}\right) \leq K_{j}=\operatorname{ord}_{<j+1}\left(\boldsymbol{T}^{\boldsymbol{n}_{0}}\right)$, whence

$$
\sum_{i>j} \operatorname{ord}_{i}\left(\boldsymbol{T}^{\boldsymbol{n}}\right)>\sum_{i>j} \operatorname{ord}_{i}\left(\boldsymbol{T}^{\boldsymbol{n}_{0}}\right)
$$


This implies that for all $\boldsymbol{T}^{\boldsymbol{n}} \in \boldsymbol{J}$ such that $\boldsymbol{n}>\boldsymbol{n}_{0}$, we must have $\prod_{i>j} T_{i}^{n_{0 j}} \nmid \boldsymbol{T}^{\boldsymbol{n}}$, i.e. $r_{\boldsymbol{n}} \boldsymbol{T}^{\boldsymbol{n}}$ is not in $P\left(T_{1}, \ldots, T_{d}\right)$. It now follows from (6-3) that

$$
\begin{aligned}
& r \frac{\boldsymbol{x}^{\boldsymbol{n}_{0}}}{\prod_{i>j} x_{i}^{n_{0 i}}}+\sum_{\substack{\boldsymbol{n} \in \boldsymbol{J}, \boldsymbol{n}_{<}<\boldsymbol{n}_{0}, \prod_{i>j} x_{i}^{n_{0 i}} \mid x^{n}}} r_{\boldsymbol{n}} \frac{\boldsymbol{x}^{\boldsymbol{n}}}{\prod_{i>j} x_{i}^{n_{0 i}}} \\
& \qquad=\frac{P\left(x_{1}, \ldots, x_{d}\right)}{\prod_{i>j} x_{i}^{n_{0 i}}} \in\left(\sum_{\substack{\boldsymbol{n} \in \boldsymbol{J} \\
\prod_{i>j} x_{i}, \mid d \boldsymbol{x}^{\boldsymbol{n}}}} r_{\boldsymbol{n}} \boldsymbol{x}^{\boldsymbol{n}}\right): \prod_{i>j} x_{i}^{n_{0 i}} .
\end{aligned}
$$

Since $R$ is generalized Cohen-Macaulay (so $\mathscr{A}(R)$ is $\mathfrak{m}$-primary), there exists a positive integer $q_{j}^{\prime}$ such that $x_{j}^{q_{j}^{\prime}} \in \mathscr{A}(R)$. Take $B=\left(x_{j+1}, \ldots, x_{d}\right)$. It can be seen that if $\boldsymbol{n}<\boldsymbol{n}_{0}$ and $\prod_{i>j} T_{i}^{n_{0 j}} \nmid \boldsymbol{T}^{\boldsymbol{n}}$ then there exists $l>j$ such that $\operatorname{ord}_{l}\left(\boldsymbol{T}^{\boldsymbol{n}}\right)>$ $\operatorname{ord}_{l}\left(\boldsymbol{T}^{\boldsymbol{n}_{0}}\right)$. On the other hand, if $\boldsymbol{n}>\boldsymbol{n}_{0}$ then it follows from (6-5) that there also exists $l>j$ such that $\operatorname{ord}_{l}\left(\boldsymbol{T}^{\boldsymbol{n}}\right)>\operatorname{ord}_{l}\left(\boldsymbol{T}^{\boldsymbol{n}_{0}}\right)$. Thus, from (6-6), we have

$$
x_{j}^{q_{j}^{\prime}}\left(r \frac{\boldsymbol{x}^{\boldsymbol{n}_{0}}}{\prod_{i>j} x_{i}^{n_{0 i}}}+\sum_{\substack{\boldsymbol{n} \in \boldsymbol{J}, \boldsymbol{n}<\boldsymbol{n}_{0}, \prod_{i>j} x_{i}^{n_{0 i}} \mid \boldsymbol{x}^{n}}} r_{\boldsymbol{n}} \frac{\boldsymbol{x}^{\boldsymbol{n}}}{\prod_{i>j} x_{i}^{n_{0 i}}}\right) \in B .
$$

Since $x_{d}, \ldots, x_{1}$ form a superficial sequence, (6-7) and Remark 3.9 imply there exists an integer $q_{j}$ such that

$$
x_{j}^{q_{j}}\left(r \frac{\boldsymbol{x}^{\boldsymbol{n}_{0}}}{\prod_{i>j} x_{i}^{n_{0 i}}}+\sum_{\substack{\boldsymbol{n} \in \boldsymbol{J}, \boldsymbol{n}<\boldsymbol{n}_{0}, \prod_{i>j} x_{i}^{n_{0 i}} \mid \boldsymbol{x}^{\boldsymbol{n}}}} r_{\boldsymbol{n}} \frac{\boldsymbol{x}^{\boldsymbol{n}}}{\prod_{i>j} x_{i}^{n_{0 i}}}\right) \in B\left(x_{1}, \ldots x_{d}\right)^{q_{j}+K+n_{0 j}-1} .
$$

Write this as

$$
r x_{1}^{n_{01}} \ldots x_{j-1}^{n_{0(j-1)}} x_{j}^{n_{0 j}+q_{j}}+\sum_{\substack{|\boldsymbol{m}|=q_{j}+K+n_{0 j}, \boldsymbol{m}<\left(n_{01}, \ldots, n_{0 j}+q_{j}, 0, \ldots, 0\right)}} u_{\boldsymbol{m}} \boldsymbol{x}^{\boldsymbol{m}}=0
$$

and let

$$
\text { (6-10) } \begin{aligned}
Q\left(T_{1}, \ldots, T_{d}\right) & =r T_{1}^{n_{01}} \ldots T_{j-1}^{n_{0(j-1)}} T_{j}^{n_{0 j}+q_{j}}+\sum_{\substack{|\boldsymbol{m}|=q_{j}+K+n_{0 j}, \boldsymbol{m}<\left(n_{01}, \ldots, n_{0 j}+q_{j}, 0, \ldots, 0\right)}} u_{\boldsymbol{m}} \boldsymbol{T}^{\boldsymbol{m}} \\
& =r \boldsymbol{T}^{\boldsymbol{m}_{0}}+\sum_{\substack{|\boldsymbol{m}|=\boldsymbol{q}_{j}+K+n_{0 j}, \boldsymbol{m}<\boldsymbol{m}_{0}}} u_{\boldsymbol{m}} \boldsymbol{T}^{\boldsymbol{m}},
\end{aligned}
$$

where $\boldsymbol{m}_{0}=\left(m_{01}, \ldots, m_{0 d}\right)=\left(n_{01}, \ldots, n_{0(j-1)}, n_{0 j}+q_{j}, 0, \ldots, 0\right)$. Then, $Q\left(T_{1}, \ldots, T_{d}\right)$ gives a new relation on $x_{1}, \ldots, x_{d}$.

Let $\boldsymbol{A}$ be the collection of all terms of $Q$ that are smaller than $r \boldsymbol{T}^{\boldsymbol{m}_{0}}$. For a term $u_{\boldsymbol{m}} \boldsymbol{T}^{\boldsymbol{m}} \in \boldsymbol{A}$, we observe the following. If $\operatorname{ord}_{i}\left(\boldsymbol{T}^{\boldsymbol{m}}\right)=0$ for all $i>j$ then 
we must have $\operatorname{ord}_{j}\left(\boldsymbol{T}^{\boldsymbol{m}}\right)>\operatorname{ord}_{i}\left(\boldsymbol{T}^{\boldsymbol{m}_{0}}\right)=m_{0 j}$, and so $\operatorname{ord}_{<j}\left(\boldsymbol{T}^{\boldsymbol{m}}\right) \leq K$. Otherwise, suppose there exists $l>j$ such that $\operatorname{ord}_{l}\left(T^{m}\right)>0$. If $\operatorname{ord}_{<j}\left(T^{m}\right)>K_{j-1}$ then from the choice of $j$, we may write $Q\left(T_{1}, \ldots, T_{d}\right)=H\left(T_{1}, \ldots, T_{d}\right) G\left(T_{1}, \ldots, T_{d}\right)+$ $Q^{\prime}\left(T_{1}, \ldots, T_{d}\right)$ where $H$ is a monomial divisible by $T_{l}\left(\operatorname{since} \operatorname{ord}_{l}\left(\boldsymbol{T}^{\boldsymbol{m}}\right)>0\right), G$ and $Q^{\prime}$ are relations on $x_{1}, \ldots, x_{d}, G$ has the form

$$
G\left(T_{1}, \ldots, T_{d}\right)=v_{\boldsymbol{k}_{0}} \boldsymbol{T}^{\boldsymbol{k}_{0}}+\sum_{\boldsymbol{k}<\boldsymbol{k}_{0},|\boldsymbol{k}|=\left|\boldsymbol{k}_{0}\right|} v_{\boldsymbol{k}} \boldsymbol{T}^{\boldsymbol{k}}
$$

with $v_{\boldsymbol{k}_{0}} \boldsymbol{T}^{\boldsymbol{k}_{0}} \mid u_{\boldsymbol{m}} \boldsymbol{T}^{\boldsymbol{m}}, \operatorname{ord}_{<j}\left(\boldsymbol{T}^{\boldsymbol{k}_{0}}\right) \leq K_{j-1}$ and $\operatorname{ord}_{j}\left(\boldsymbol{T}^{\boldsymbol{k}_{0}}\right)=\cdots=\operatorname{ord}_{d}\left(\boldsymbol{T}^{\boldsymbol{k}_{0}}\right)=0$. Notice that since $H$ is divisible by $T_{l}$, all terms in $H\left(T_{1}, \ldots, T_{d}\right) G\left(T_{1}, \ldots, T_{d}\right)$ are smaller than $r \boldsymbol{T}^{\boldsymbol{m}_{0}}$. By replacing $Q$ by $Q^{\prime}$ and repeating the process to successively remove all terms $u_{\boldsymbol{m}} \boldsymbol{T}^{\boldsymbol{m}} \in \boldsymbol{A}$ such that $\operatorname{ord}_{<j}\left(\boldsymbol{T}^{\boldsymbol{m}}\right)>K_{j-1}$ from $Q$, we may assume that in our relation $Q\left(T_{1}, \ldots, T_{d}\right)$, every term $u_{m} \boldsymbol{T}^{m}$ satisfies the condition $\operatorname{ord}_{<j}\left(\boldsymbol{T}^{\boldsymbol{m}}\right) \leq K_{j-1}$.

Let $\gamma \in \mathscr{A}(R)$ be a homology multiplier such that $\left(\gamma, x_{1}, \ldots, \widehat{x_{j}}, \ldots, x_{d}\right)$ is an s.o.p. Since $\gamma$ is part of an s.o.p. and $H_{\mathfrak{m}}^{0}(R)=0, \gamma$ is not a zero-divisor. Let $\boldsymbol{M}$ denote the set of all monomials appearing in $Q$. Recall that $K=\sum_{i<j} n_{0 i}=$ $\sum_{i<j} m_{0 i} \leq K_{j-1}$ and $B=\left(x_{j+1}, \ldots, x_{d}\right)$.

Let $F_{1}\left(T_{1}, \ldots, T_{d}\right)$ be the sum of all terms of $Q$ that are divisible by $T_{j}^{m_{0 j}-K_{j-1}}$ :

$$
F_{1}\left(T_{1}, \ldots, T_{d}\right)=T_{j}^{m_{0 j}-K_{j-1}}\left(r \frac{\boldsymbol{T}^{\boldsymbol{m}_{0}}}{T_{j}^{m_{0 j}-K_{j-1}}}+\sum_{\substack{\boldsymbol{m} \in \boldsymbol{M}, \boldsymbol{m}<\boldsymbol{m}_{0}, T_{j}^{m_{0 j}-K_{j-1}} \mid \boldsymbol{T}^{\boldsymbol{m}}}} u_{\boldsymbol{m}} \frac{\boldsymbol{T}^{\boldsymbol{m}}}{T_{j}^{m_{0 j}-K_{j-1}}}\right) .
$$

Let $G_{1}\left(T_{1}, \ldots, T_{d}\right)=\frac{F_{1}\left(T_{1}, \ldots, T_{d}\right)}{T_{j}^{m_{0 j}-K_{j-1}}}$. Then, (6-9) gives

$G_{1}\left(x_{1}, \ldots, x_{d}\right) \in\left(\boldsymbol{x}^{\boldsymbol{m}} \in \boldsymbol{M} \mid \boldsymbol{m}<\boldsymbol{m}_{0}, \operatorname{ord}_{j}\left(\boldsymbol{x}^{\boldsymbol{m}}\right)<m_{0 j}-K_{j-1}\right): x_{j}^{m_{0 j}-K_{j-1}}$

$$
\begin{aligned}
=\left(x^{\boldsymbol{m}} \in \boldsymbol{M} \mid \operatorname{ord}_{j}\left(\boldsymbol{x}^{\boldsymbol{m}}\right)<m_{0 j}-K_{j-1}\right. \text { and } \\
\left.\qquad \sum_{i>j} \operatorname{ord}_{i}\left(\boldsymbol{x}^{\boldsymbol{m}}\right)>K\right): x_{j}^{m_{0 j}-K_{j-1}} .
\end{aligned}
$$

This is because

$$
\begin{aligned}
\sum_{i>j} \operatorname{ord}_{i}\left(\boldsymbol{x}^{\boldsymbol{m}}\right) & >\sum_{i=1}^{d} \operatorname{ord}_{i}\left(\boldsymbol{x}^{\boldsymbol{m}}\right)-\operatorname{ord}_{<j}\left(\boldsymbol{x}^{\boldsymbol{m}}\right)-\left(m_{0 j}-K_{j-1}\right) \\
& =\sum_{i=1}^{d} \operatorname{ord}_{i}\left(\boldsymbol{x}^{\boldsymbol{m}_{0}}\right)-\operatorname{ord}_{<j}\left(\boldsymbol{x}^{\boldsymbol{m}}\right)-\left(m_{0 j}-K_{j-1}\right) \\
& \geq K+m_{0 j}-K_{j-1}-\left(m_{0 j}-K_{j-1}\right)=K .
\end{aligned}
$$

Therefore, since $\gamma$ is a homology multiplier, we have

$$
\gamma G_{1}\left(x_{1}, \ldots, x_{d}\right) \in\left(x_{1}, \ldots, \widehat{x_{j}}, \ldots, x_{d}\right)^{K_{j-1}} B^{K+1} .
$$


Similarly, let $F_{2}\left(T_{1}, \ldots, T_{d}\right)$ be the sum of all terms of $Q$ that are divisible by $T_{j}^{m_{0 j}-K_{j-1}-1}$, i.e.,

$$
F_{2}\left(T_{1}, \ldots, T_{d}\right)=T_{j}^{m_{0 j}-K_{j-1}-1}\left(r \frac{\boldsymbol{T}^{\boldsymbol{m}_{0}}}{T_{j}^{m_{0 j}-K_{j-1}-1}}+\sum_{\substack{\boldsymbol{m} \in \boldsymbol{M}, \boldsymbol{m}<\boldsymbol{m}_{0}, T_{j}^{m_{0 j}-K_{j-1}-1} \mid \boldsymbol{T}^{\boldsymbol{m}}}} u_{\boldsymbol{m}} \frac{\boldsymbol{T}^{\boldsymbol{m}}}{T_{j}^{m_{0 j}-K_{j-1}-1}}\right),
$$

and let $G_{2}\left(T_{1}, \ldots, T_{d}\right)=\frac{F_{2}\left(T_{1}, \ldots, T_{d}\right)}{T_{j}^{m_{0 j}-K_{j-1}-1}}$, we then have

$G_{2}\left(x_{1}, \ldots, x_{d}\right) \in\left(\boldsymbol{x}^{\boldsymbol{m}} \in \boldsymbol{M} \mid \boldsymbol{m}<\boldsymbol{m}_{0}, \operatorname{ord}_{j}\left(\boldsymbol{x}^{\boldsymbol{m}}\right)<m_{0 j}-K_{j-1}-1\right): x_{j}^{m_{0 j}-K_{j-1}-1}$

This is because

$$
\begin{aligned}
=\left(\boldsymbol{x}^{\boldsymbol{m}} \in \boldsymbol{M} \mid \operatorname{ord}_{j}\left(\boldsymbol{x}^{\boldsymbol{m}}\right)<\right. & m_{0 j}-K_{j-1}-1 \text { and } \\
& \left.\sum_{i>j} \operatorname{ord}_{i}\left(\boldsymbol{x}^{\boldsymbol{m}}\right)>K+1\right): x_{j}^{m_{0 j}-K_{j-1}-1} .
\end{aligned}
$$

$$
\begin{aligned}
\sum_{i>j} \operatorname{ord}_{i}\left(x^{m}\right) & >\sum_{i=1}^{d} \operatorname{ord}_{i}\left(x^{m}\right)-\operatorname{ord}_{<j}\left(x^{m}\right)-\left(m_{0 j}-K_{j-1}-1\right) \\
& =\sum_{i=1}^{d} \operatorname{ord}_{i}\left(\boldsymbol{x}^{\boldsymbol{m}_{0}}\right)-\operatorname{ord}_{<j}\left(\boldsymbol{x}^{\boldsymbol{m}}\right)-\left(m_{0 j}-K_{j-1}-1\right) \\
& \geq K+m_{0 j}-K_{j-1}-\left(m_{0 j}-K_{j-1}-1\right)=K+1 .
\end{aligned}
$$

Thus, again since $\gamma$ is a homology multiplier, we get

$$
\gamma G_{2}\left(x_{1}, \ldots, x_{d}\right) \in\left(x_{1}, \ldots, \widehat{x_{j}}, \ldots, x_{d}\right)^{K_{j-1}} B^{K+2} .
$$

Proceeding in this way, we obtain:

$$
\begin{aligned}
\gamma G_{1}\left(x_{1}, \ldots, x_{d}\right) & \in\left(x_{1}, \ldots, \widehat{x_{j}}, \ldots, x_{d}\right)^{K_{j-1}} B^{K+1}, \\
\gamma G_{2}\left(x_{1}, \ldots, x_{d}\right) & \in\left(x_{1}, \ldots, \widehat{x_{j}}, \ldots, x_{d}\right)^{K_{j-1}} B^{K+2}, \quad \ldots, \\
\gamma G_{p}\left(x_{1}, \ldots, x_{d}\right) & \in\left(x_{1}, \ldots, \widehat{x_{j}}, \ldots, x_{d}\right)^{K_{j-1}} B^{K+p}, \quad \ldots, \\
\gamma G_{m_{0 j}-K_{j-1}+1}\left(x_{1}, \ldots, x_{d}\right) & \in\left(x_{1}, \ldots, \widehat{x_{j}}, \ldots, x_{d}\right)^{K_{j-1}} B^{K+m_{0 j}-K_{j-1}+1},
\end{aligned}
$$

where

$G_{p}\left(T_{1}, \ldots, T_{d}\right)=\frac{F_{p}\left(T_{1}, \ldots, T_{d}\right)}{T_{j}^{m_{0 j}-K_{j-1}-p+1}}=r T_{1}^{m_{01}} \ldots T_{j-1}^{m_{0(j-1)}} T_{j}^{K_{j-1}+p-1}+$ smaller terms and $F_{p}\left(T_{1}, \ldots, T_{d}\right)$ is the sum of all terms in $Q$ that are divisible by $T_{j}^{m_{0 j}-K_{j-1}-p+1}$, for $1 \leq p \leq m_{0 j}-K_{j-1}+1$.

Observe that $G_{p}\left(x_{1}, \ldots, x_{d}\right)$ has degree $K_{j-1}+K+p-1$ in $x_{1}, \ldots, \widehat{x_{j}}, \ldots, x_{d}$. Let $C_{p}=\left(x_{1}, \ldots, \widehat{x_{j}}, \ldots, x_{d}\right)^{K_{j-1}} B^{K+p}$. It follows from the relations (6-12) that, for each $1 \leq p \leq m_{0 j}-K_{j-1}+1$, we can write $\gamma G_{p}\left(x_{1}, \ldots, x_{d}\right)=H_{p}\left(x_{1}, \ldots, x_{d}\right)$, where $H_{p}\left(x_{1}, \ldots, x_{d}\right) \in C_{p}$ is a polynomial of degree $K_{j-1}+K+p$ on $x_{1}, \ldots, \widehat{x_{j}}$, $\ldots, x_{d}$. From now on, we shall write $G_{p}$ for $G_{p}\left(x_{1}, \ldots, x_{d}\right)$, and likewise for $H_{p}$. 
We order the terms in $H_{p}$ with respect to our monomial ordering (graded reverse lex), and let $s_{p}$ be the leading coefficient of $H_{p}$. That is,

$$
\begin{aligned}
\gamma G_{1} & =s_{1} \boldsymbol{x}^{\boldsymbol{m}_{1}}+\sum_{\boldsymbol{m}<\boldsymbol{m}_{1}} s_{\boldsymbol{m}} \boldsymbol{x}^{\boldsymbol{m}}, \\
\gamma G_{2} & =s_{2} \boldsymbol{x}^{\boldsymbol{m}_{2}}+\sum_{\boldsymbol{m}<\boldsymbol{m}_{2}} s_{\boldsymbol{m}} \boldsymbol{x}^{\boldsymbol{m}}, \quad \ldots, \\
\gamma G_{p} & =s_{p} \boldsymbol{x}^{\boldsymbol{m}_{p}}+\sum_{\boldsymbol{m}<\boldsymbol{m}_{p}} s_{\boldsymbol{m}} \boldsymbol{x}^{\boldsymbol{m}}, \quad \ldots, \\
\gamma G_{m_{0 j}-K_{j-1}+1} & =s_{m_{0 j}-K_{j-1}+1} \boldsymbol{x}^{\boldsymbol{m}_{m_{0 j}-K_{j-1}+1}}+\sum_{\boldsymbol{m}<\boldsymbol{m}_{m_{0 j}-K_{j-1}+1}} s_{\boldsymbol{m}} \boldsymbol{x}^{\boldsymbol{m}},
\end{aligned}
$$

where $\boldsymbol{x}^{\boldsymbol{m}_{p}} \in C_{p}$ and $\left|\boldsymbol{m}_{p}\right|=K_{j-1}+K+p$. In the $p$-th equality of (6-13), among all different ways of writing $\gamma G_{p}=H_{p}$ where $G_{p}$ is a polynomial expression in $x_{1}, \ldots, x_{d}$ of degree $K_{j-1}+K+p-1$ and leading term $r x_{1}^{m_{01}} \ldots x_{j-1}^{m_{0(j-1)}} x_{j}^{K_{j-1}+p-1}$ and $H_{p}$ is a polynomial expression in $x_{1}, \ldots, x_{d}$ of degree $K_{j-1}+K+p$, we choose the one with smallest possible leading term $s_{p} x^{m_{p}}$ on the right-hand side.

Claim 6.5. There exists an integer $p \leq N_{j}$ such that $H_{p}=0$.

Proof of Claim. From (6-4) we have $m_{0 j}-K_{j-1}+1 \geq n_{0 j}-K_{j-1}+1 \geq N_{j}+1$. By contradiction, suppose the assertion is false. That is, $s_{1}, \ldots, s_{N_{j}}$ are all nonzero. Fix an integer $1 \leq p \leq m_{0 j}-K_{j-1}+1$, and suppose the $x_{i}$ 's that appear in $\boldsymbol{x}^{\boldsymbol{m}_{p}}$ are in $\left\{x_{i} \mid i \geq h\right\} \backslash\left\{x_{j}\right\}$ (and $h$ is chosen to be the largest integer with this property). Then

$$
\begin{aligned}
s_{p} & \in\left(\gamma,\left\{\boldsymbol{x}^{\boldsymbol{m}} \in C_{p} \mid \boldsymbol{m}<\boldsymbol{m}_{p}\right\}\right): \boldsymbol{x}^{\boldsymbol{m}_{p}} \\
& =\left(\gamma,\left\{\boldsymbol{x}^{\boldsymbol{m}} \in C_{p} \mid \operatorname{ord}_{i}\left(\boldsymbol{x}^{\boldsymbol{m}}\right)>\operatorname{ord}_{i}\left(\boldsymbol{x}^{\boldsymbol{m}_{p}}\right) \text { for some } i \geq h+1\right\}\right): \boldsymbol{x}^{\boldsymbol{m}_{p}} .
\end{aligned}
$$

Choose $\alpha \in \mathscr{A}(R)$ such that $\left(\gamma,\left\{x_{h+1}, \ldots, x_{d}\right\} \backslash\left\{x_{j}\right\}, \alpha\right)$ is part of an s.o.p. (since $x_{h}$ divides $\boldsymbol{x}^{\boldsymbol{m}_{p}}$, we have $h \neq j$, so this choice is possible). Then,

$$
\alpha s_{p} \in\left(\gamma,\left\{x_{h+1}, \ldots, x_{d}\right\} \backslash\left\{x_{j}\right\}\right), \quad \text { that is, } s_{p} \in\left(\gamma,\left\{x_{h+1}, \ldots, x_{d}\right\} \backslash\left\{x_{j}\right\}\right): \alpha .
$$

Since $R$ is a generalized Cohen-Macaulay ring and $\left(\gamma,\left\{x_{h+1}, \ldots, x_{d}\right\} \backslash\left\{x_{j}\right\}, \alpha\right)$ is part of an s.o.p., $\alpha$ avoids all associated primes of $\left(\gamma,\left\{x_{h+1}, \ldots, x_{d}\right\} \backslash\left\{x_{j}\right\}\right)$ except possibly $\mathfrak{m}$. Hence, we have

$$
\bar{s}_{p} \in H_{\mathfrak{m}}^{0}\left(R /\left(\gamma,\left\{x_{h+1}, \ldots, x_{d}\right\} \backslash\left\{x_{j}\right\}\right)\right),
$$

where $\bar{s}_{p}$ denotes the image of $s_{p}$ in $R /\left(\gamma,\left\{x_{h+1}, \ldots, x_{d}\right\} \backslash\left\{x_{j}\right\}\right)$.

Consider the sequence $\left\{\boldsymbol{m}_{1}, \boldsymbol{m}_{2}, \ldots, \boldsymbol{m}_{N_{j}}\right\}$. Since

$$
N_{j}=M\left(d, 2 K_{j-1}, M_{j}(L-1)+1\right) \geq M\left(d, K_{j-1}+K, M_{j}(L-1)+1\right)
$$

and $\left|\boldsymbol{m}_{1}\right|=K_{j-1}+K+1$, it follows from Lemma 5.2 that there exists an increasing subsequence of length $V=M_{j}(L-1)+1$, say $\boldsymbol{m}_{l_{1}} \preceq \boldsymbol{m}_{l_{2}} \preceq \cdots \preceq \boldsymbol{m}_{l_{V}}$, with $1 \leq$ $l_{1}<l_{2}<\cdots<l_{V} \leq N_{j} \leq m_{0 j}-K_{j-1}$. Since $C_{p}=\left(x_{1}, \ldots, \widehat{x_{j}}, \ldots, x_{d}\right)^{K_{j-1}} B^{K+p}$, 
we have $\operatorname{ord}_{<j}\left(\boldsymbol{x}^{\boldsymbol{m}_{p}}\right) \leq K_{j-1}$ for any $1 \leq p \leq m_{0 j}-K_{j-1}+1$. Thus, since $M_{j}$ is the number of $(j-1)$-tuples of nonnegative integers whose sum is at most $K_{j-1}$, we can choose from the sequence $\boldsymbol{m}_{l_{1}} \preceq \boldsymbol{m}_{l_{2}} \preceq \cdots \preceq \boldsymbol{m}_{l_{V}}$ a subsequence of length $L, \boldsymbol{m}_{i_{1}} \preceq \boldsymbol{m}_{i_{2}} \preceq \cdots \preceq \boldsymbol{m}_{i_{L}}$ such that they have the same $j-1$ first components, i.e., for any $1 \leq a, b \leq L$ we have $\left(\operatorname{ord}_{1}\left(\boldsymbol{x}^{\boldsymbol{m}_{i_{a}}}\right), \ldots, \operatorname{ord}_{j-1}\left(\boldsymbol{x}^{\boldsymbol{m}_{i_{a}}}\right)\right)=$ $\left(\operatorname{ord}_{1}\left(\boldsymbol{x}^{\boldsymbol{m}_{i_{b}}}\right), \ldots, \operatorname{ord}_{j-1}\left(\boldsymbol{x}^{\boldsymbol{m}_{i_{b}}}\right)\right)$. Since $L=\left(L_{2}-1\right)+\cdots+\left(L_{d}-1\right)+1$, we can choose from this sequence yet another subsequence $\boldsymbol{m}_{p_{1}} \preceq \boldsymbol{m}_{p_{2}} \preceq \cdots \preceq \boldsymbol{m}_{p_{L q}}$, for some $2 \leq q \leq d$, such that

$$
\bar{s}_{p_{t}} \in H_{\mathfrak{m}}^{0}\left(R /\left(\gamma,\left\{x_{q+1}, \ldots, x_{d}\right\} \backslash\left\{x_{j}\right\}\right)\right),
$$

for any $t=1,2, \ldots, L_{q}$. It follows from Remark 6.3 that

$$
\lambda\left(H_{\mathfrak{m}}^{0}\left(R /\left(\gamma,\left\{x_{q+1}, \ldots, x_{d}\right\} \backslash\left\{x_{j}\right\}\right)\right)\right)<L_{q},
$$

so we can write

$$
s_{p_{L_{q}}}=\sum_{i>q, i \neq j} a_{i} x_{i}+a_{j} \gamma+\sum_{i=1}^{L_{q}-1} b_{i} s_{p_{i}} .
$$

Substitute this into the equality $\gamma G_{p_{L_{q}}}=H_{p_{L_{q}}}$ to get

(6-14) $\gamma\left(G_{p_{L_{q}}}-a_{j} x^{\boldsymbol{m}_{p_{L_{q}}}}\right)=\sum_{\substack{i>q, i \neq j}} a_{i} x_{i} x^{\boldsymbol{m}_{p_{L_{q}}}}+\sum_{i=1}^{L_{q}-1} b_{i} s_{p_{i}} x^{\boldsymbol{m}_{p_{L_{q}}}}+\sum_{\boldsymbol{m}<\boldsymbol{m}_{p_{L_{q}}}} s_{\boldsymbol{m}} \boldsymbol{x}^{\boldsymbol{m}}$

$$
\begin{aligned}
=\sum_{\substack{i>q, i \neq j}}\left(a_{i} x_{q}\right) \frac{x^{\boldsymbol{m}_{p_{L_{q}}}} x_{i}}{x_{q}}+\sum_{i=1}^{L_{q}-1}\left(b_{i} \frac{\boldsymbol{x}^{\boldsymbol{m}_{p_{L_{q}}}}}{\boldsymbol{x}^{\boldsymbol{m}_{p_{i}}}}\right) s_{p_{i}} \boldsymbol{x}^{\boldsymbol{m}_{p_{i}}} \\
+\sum_{\boldsymbol{m}<\boldsymbol{m}_{p_{L_{q}}}} s_{\boldsymbol{m}} \boldsymbol{x}^{\boldsymbol{m}} .
\end{aligned}
$$

Since $\boldsymbol{x}^{\boldsymbol{m}_{p_{L_{q}}}} \in C_{p_{L_{q}}}$, we have (after absorbing extra powers into the coefficient if necessary) $a_{j} x^{m_{p_{L q}}}<r x_{1}^{m_{01}} \ldots x_{j-1}^{m_{0(j-1)}} x_{j}^{K_{j-1}+p_{L_{q}}-1}$. Also,

$$
\frac{x^{m_{p_{L_{q}}}} x_{i}}{x_{q}}<\boldsymbol{x}^{\boldsymbol{m}_{p_{L_{q}}}} \text { for } i>q .
$$

Furthermore, since $\boldsymbol{m}_{p_{L_{q}}}$ and $\boldsymbol{m}_{p_{i}}$ have the same $j-1$ first components, we have

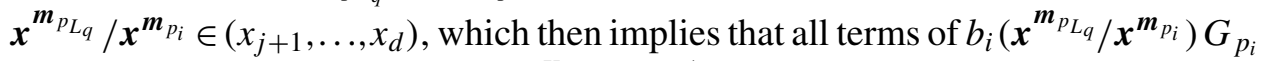
are smaller than $r x_{1}^{m_{01}} \ldots x_{j-1}^{m_{0(j-1)}} x_{j}^{K_{j-1}+p_{L_{q}-1}}$. Thus, after using (6-13) to replace $s_{p_{i}} \boldsymbol{x}^{\boldsymbol{m}_{p_{i}}}$ by $\left(\gamma G_{p_{i}}-\sum_{|\boldsymbol{m}|=\left|\boldsymbol{m}_{p_{i}}\right|, \boldsymbol{m}<\boldsymbol{m}_{p_{i}}} s_{\boldsymbol{m}} x^{\boldsymbol{m}}\right)$ in (6-14) and moving terms involving $\gamma$ to the left-hand side, we get a relation

$$
\gamma\left(G_{p_{L_{q}}}+\text { terms smaller than } r x_{1}^{m_{01}} \ldots x_{j-1}^{m_{0(j-1)}} x_{j}^{K_{j-1}+p_{L_{q}}-1}\right)=H_{p_{L_{q}}}^{\prime} .
$$


That is,

$$
\gamma\left(r x_{1}^{m_{01}} \ldots x_{j-1}^{m_{0(j-1)}} x_{j}^{K_{j-1}+p_{L_{q}-1}}+\text { smaller terms }\right)=H_{p_{L_{q}}}^{\prime},
$$

where the leading term of $H_{p_{L_{q}}}^{\prime}$ is strictly smaller than $s_{p_{L_{q}}} \boldsymbol{x}^{\boldsymbol{m}_{p_{L_{q}}}}$, which is the leading term of $H_{p_{L q}}$. This contradicts the way (6-13) was chosen. Our claim is proved.

We have just shown that there exists an integer $p \leq N_{j}$ such that the $p$-th equality in (6-13) is $\gamma G_{p}=0$. Thus, since $\gamma$ is not a zero-divisor, $G_{p}=0$. That is,

$$
r x_{1}^{m_{01}} \ldots x_{j-1}^{m_{0(j-1)}} x_{j}^{K_{j-1}+p-1}+\text { smaller terms }=0 .
$$

This gives a relation on $x_{1}, \ldots, x_{d}$, which by abusing language we shall denote by $G_{p}$ :

$$
\begin{aligned}
G_{p}\left(T_{1}, \ldots, T_{d}\right) & =r T_{1}^{m_{01}} \ldots T_{j-1}^{m_{0(j-1)}} T_{j}^{K_{j-1}+p-1}+\text { smaller terms } \\
& =r T_{1}^{n_{01}} \ldots T_{j-1}^{n_{0(j-1)}} T_{j}^{K_{j-1}+p-1}+\text { smaller terms. }
\end{aligned}
$$

Observe that $K_{j-1}+p-1 \leq K_{j-1}+N_{j}-1<n_{0 j}$ by (6-4). We now can write

$$
F\left(T_{1}, \ldots, T_{d}\right)=\left[T_{j}^{n_{0 j}-K_{j-1}-p+1} \prod_{i>j} T_{i}^{n_{0 i}}\right] G_{p}\left(T_{1}, \ldots, T_{d}\right)+F^{\prime}\left(T_{1}, \ldots, T_{d}\right),
$$

where both $G_{p}$ and $F^{\prime}$ are relations on $x_{1}, \ldots, x_{d}$. It is clear that every term $r_{\boldsymbol{n}} \boldsymbol{T}^{\boldsymbol{n}}$ of $F^{\prime}$ with ord ${ }_{<j+1}\left(\boldsymbol{T}^{\boldsymbol{n}}\right)>K_{j}$ is smaller than $r_{\boldsymbol{n}_{0}} \boldsymbol{T}^{\boldsymbol{n}_{0}}$. We obtain a contradiction. Hence, the theorem is proved.

\section{Relation type in rings with non-Cohen-Macaulay locus of dimension one}

This section is devoted to treating the unknown situation where the ring $R$ has non-CM locus of dimension 1. Our main theorem shows that $R$ satisfies bounded relation type provided $\mathscr{A}(\hat{R})$ is a prime ideal in the completion $\hat{R}$ of $R$. As before, throughout the section, $(R, \mathfrak{m}, k)$ shall denote a local ring $R$ with maximal ideal $\mathfrak{m}$ and residue field $k$.

Lemma 7.1. Let $(R, \mathfrak{m}, k)$ be a complete unmixed local ring of dimension $d \geq 3$. Assume that $\mathscr{A}(R)=P$ is a dimension one prime. Then $\lambda\left(H_{\mathfrak{m}}^{1}(R)\right)<\infty$. Let $x_{d} \in R$ be a non-zero-divisor such that its image in $R / P$ is in $\mathfrak{m} R / P-\mathfrak{m}^{\mathfrak{r}} R / P$ for some positive integer $\mathfrak{r}$. Then for any system of parameters $x_{1}, \ldots, x_{d}$ and any integer $1<j \leq d$ there is a bound on $\lambda\left(H_{\mathfrak{m}}^{0}\left(R /\left(x_{j}, \ldots, x_{d}\right)\right)\right)$, depending only on $\mathfrak{r}$ and $j$.

Proof. The assertion that $\lambda\left(H_{\mathfrak{m}}^{1}(R)\right)<\infty$ follows by duality. Let $S \subseteq R$ be a Gorenstein ring with $R$ module-finite over $S$. Then $H_{\mathfrak{m}}^{1}(R)$ is dual to $\operatorname{Ext}_{S}^{d-1}(R, S)$. For any nonmaximal prime $Q \subseteq R$ of height $h$, let $q=Q \cap S$. Then $\left(\operatorname{Ext}_{S}^{d-1}(R, S)\right)_{Q}=$ 
$\operatorname{Ext}_{S_{q}}^{d-1}\left(R_{Q}, S_{q}\right)$ is dual to $H_{Q R_{Q}}^{h-d+1}\left(R_{Q}\right)$. If $h<d-1$ this clearly vanishes. Otherwise $h=d-1$, and the assumption that $R$ is unmixed gives $H_{Q R_{Q}}^{0}\left(R_{Q}\right)=0$. Thus $\lambda\left(H_{\mathfrak{m}}^{1}(R)\right)=\lambda\left(\operatorname{Ext}_{S}^{d-1}(R, S)\right)<\infty$.

For simplicity of notation let $x_{d}=x$. We next observe that the hypotheses give the non-CM locus of $R$ is $\{P, \mathfrak{m}\}$. Since $x \notin P$, the ring $R / x R$ is generalized CM. Thus, if we can bound the lengths of $H_{\mathfrak{m}}^{i}(R / x R)$ for $0 \leq i \leq d-2$, depending only on $i$ and $\mathfrak{r}$ then Schenzel's result (Lemma 6.2) may be applied.

Since $P=\mathscr{A}(R)$ annihilates $H_{\mathfrak{m}}^{i}(R)$ for $0 \leq i \leq d-1$, we see that $H_{\mathfrak{m}}^{i}(R)$ is an Artinian $R / P$ module. Let $E=E_{R / P}(R / \mathfrak{m})$ be an injective hull. For each $1<i<d$ there is an exact sequence $0 \rightarrow H_{\mathfrak{m}}^{i}(R) \rightarrow E^{t_{i}} \rightarrow C_{i} \rightarrow 0$ (and $C_{i}$ is Artinian). From the snake lemma applied to multiplication of this short exact sequence by $x$ we get an exact sequence

$0 \rightarrow \operatorname{Ann}_{H_{\mathfrak{m}}^{i}(R)} x \rightarrow\left(\operatorname{Ann}_{E} x\right)^{t_{i}} \rightarrow \operatorname{Ann}_{C_{i}} x \rightarrow H_{\mathfrak{m}}^{i}(R) / x H_{\mathfrak{m}}^{i}(R) \rightarrow E^{t_{i}} / x E^{t_{i}}=0$.

We also have, from the sequence $0 \rightarrow R \stackrel{x}{\rightarrow} R \rightarrow R / x R \rightarrow 0$, the long exact sequence in local cohomology, which gives

$$
0 \rightarrow H_{\mathfrak{m}}^{i}(R) / x H_{\mathfrak{m}}^{i}(R) \rightarrow H_{\mathfrak{m}}^{i}(R / x R) \rightarrow \operatorname{Ann}_{H_{\mathfrak{m}}^{i+1}(R)} x \rightarrow 0 .
$$

From (7-1) and (7-2) we see that it suffices to bound $\lambda\left(H_{\mathfrak{m}}^{i}(R) / x H_{\mathfrak{m}}^{i}(R)\right)$ and $\lambda\left(\operatorname{Ann}_{H_{\mathfrak{m}}^{i+1}(R)} x\right)$ for $i \leq d-2$, and hence to bound $\lambda\left(\operatorname{Ann}_{E} x\right)$ depending only on $\mathfrak{r}$ (using the fact that each $C_{i}$ embeds in a finite direct sum of $E$ 's). By duality, $\lambda\left(\operatorname{Ann}_{E} x\right)=\lambda(R /(P+x R))$.

Thus the problem reduces to showing that if $(S, \mathfrak{m})$ is a one dimensional complete domain and $x \in \mathfrak{m}-\mathfrak{m}^{\mathfrak{r}}$ then there is a bound on $\lambda(S / x S)$ which depends only on r. Let $T$ be the integral closure of $S$, and set $N$ to be the degree of the extension of fraction fields. Then $\lambda_{S}(S / x S) \leq N \lambda_{T}(T / x T)$. By Rees's strong valuation theorem, there is an integer $k$ such that $\operatorname{ord}_{T}(x) \leq \operatorname{ord}_{S}(x)+k$ [Rees 1988]. This shows that $\lambda_{S}(S / x S) \leq N(\mathfrak{r}+k)$.

Theorem 7.2. Let $(R, \mathfrak{m}, k)$ be a formally unmixed local ring of dimension $d$ such that $\mathscr{A}(\hat{R})$ is a prime ideal of dimension one in the completion $\hat{R}$ of $R$. Then $R$ has a uniform bound on relation type of parameter ideals.

Proof. By [Wang 1997b, Lemma 4.1], since our hypotheses pass to the completion, we may assume that $R$ is complete. By [Wang 1997a, Lemma 2.2], we may also assume that $H_{\mathfrak{m}}^{0}(R)=0$. Observe further that we can assume $k$ is infinite. Indeed, let $S=R[y]_{m R[y]}$. Then the residue field of $S$ is infinite. Since $R \hookrightarrow S$ is smooth, for $c \in R, R_{c}$ is CM if and only if $S_{c}$ is CM. Thus, $\mathscr{A}(R) S \subseteq \mathscr{A}(S)$. Moreover, for any prime ideal $P \subseteq R, P S$ is prime. This implies that if $\mathscr{A}(S)$ properly contains $\mathscr{A}(R) S$ then it is primary to the maximal ideal of $S$, and so $S$ has f.l.c. It then 
follows that $R$ has f.l.c., a contradiction. Hence, $\mathscr{A}(R) S=\mathscr{A}(S)$. We may pass from $R$ to $S$ and assume that $k$ is infinite.

Let $I=\left(x_{1}, \ldots, x_{d}\right)$ be a parameter ideal in $R$. We may pick $x_{d}, x_{d-1}, \ldots, x_{1}$ to form a superficial sequence. If $d=2$, the theorem is true by [Wang 1997b].

Suppose $d \geq 3$. Since $\operatorname{dim} R / \mathscr{A}(R)=1$, in $R / \mathscr{A}(R), \mathfrak{m}+\mathscr{A}(R)$ has a principal minimal reduction $\bar{w}$. Let $\mathfrak{r}$ be the reduction number of $\mathfrak{m}+\mathscr{A}(R)$ in $R / \mathscr{A}(R)$, and let $w$ be a representative of $\bar{w}$ in $R$. Suppose $y \in R$. Then, there exists an integer $t_{y} \geq 0$ such that $\bar{y} \in \overline{\mathfrak{m}}^{t_{y}}-\overline{\mathfrak{m}}^{t_{y}-1}$ in $R / \mathscr{A}(R)$. Observe that if $t_{y} \geq \mathfrak{r}+1$, then we can write $y=y^{\prime} w^{l}+\alpha$ where $y^{\prime} \in \mathfrak{m}^{\mathfrak{r}}, l=t_{y}-\mathfrak{r}$ and $\alpha \in \mathscr{A}(R)$. If $t_{y} \leq \mathfrak{r}$, we can write $y$ in the same form $y=y^{\prime} w^{l}+\alpha$ by letting $y^{\prime}=y, l=0$ and $\alpha=0$.

By replacing $x_{d}$ by a generic combination of $x_{1}, \ldots, x_{d-1}$, we may first assume that $t_{x_{d}}=\min \left\{t_{x_{i}} \mid 1 \leq i \leq d\right\}$ (since being superficial is an open condition, after replacing $x_{d}$ by a generic combination of $x_{1}, \ldots, x_{d-1}$, the sequence $x_{d}, x_{d-1}, \ldots, x_{1}$ is still superficial). We will now use Theorem 4.3 to modify our parameter ideal as follows. If $t_{x_{d}} \geq \mathfrak{r}+1$ then we write $x_{d}=x_{d}^{\prime} w^{t}+\alpha$ where $x_{d}^{\prime} \in \mathfrak{m}^{\mathfrak{r}}, t=t_{x_{d}}-\mathfrak{r}$ and $\alpha \in \mathscr{A}(R)$. We may choose $w, x_{d}^{\prime}$ and $\alpha$ such that $\left(x_{1}, \ldots, x_{d-1}, w\right)$ and $\left(x_{1}, \ldots, x_{d-1}, x_{d}^{\prime}\right)$ are s.o.p.'s. That is, $\left(x_{1}, \ldots, x_{d-1}, x_{d}^{\prime} w^{t}\right)$ is an s.o.p. Let $y_{i}=x_{i}$ for $1 \leq i \leq d-1$, and $y_{d}=x_{d}^{\prime} w^{t}$. It follows from Theorem 4.3 that

$$
\operatorname{rt}\left(x_{1}, \ldots, x_{d}\right)=\operatorname{rt}\left(y_{1}, \ldots, y_{d}\right) .
$$

If $t_{x_{d}} \geq \mathfrak{r}$, then we let $\left(y_{1}, \ldots, y_{d}\right)=\left(x_{1}, \ldots, x_{d}\right)$ and $x_{d}^{\prime}=x_{d}$. The theorem will be proved if we can show that $\operatorname{rt}\left(y_{1}, y_{2}, \ldots, y_{d}\right)$ is uniformly bounded. By Lemma 4.5 , we may assume that $y_{d}, \ldots, y_{1}$ form a superficial sequence.

It follows from Lemma 7.1 that for $2 \leq q \leq d$ there exists a uniform bound $B_{q}$ (depending only on $q$ and $\mathfrak{r}$ ) with the following property: if $z_{q-1}, \ldots, z_{d-1} \in R$ are such that $\left(z_{q}, \ldots, z_{d-1}, x_{d}^{\prime}\right)$ is part of an s.o.p., then

$$
\lambda\left(H_{\mathfrak{m}}^{0}\left(R /\left(z_{q-1}, \ldots, z_{d-1}, x_{d}^{\prime}\right)\right)\right)<B_{q} .
$$

For $q=2, \ldots, d$, set

$$
L_{q}=\max \left\{B_{q}, B_{q+1}\right\} .
$$

By considering the exact sequence $0 \rightarrow R \stackrel{\gamma}{\rightarrow} R \rightarrow R /(\gamma) \rightarrow 0$, for any non-zerodivisor $\gamma \in \mathscr{A}(R)$ which is part of an s.o.p., it also follows from Lemma 7.1 that there exists a uniform bound $L_{d+1}$ not depending on $\gamma$ such that

$$
\lambda\left(H_{\mathfrak{m}}^{0}(R /(\gamma))\right)<L_{d+1} .
$$

Let $L=\left(L_{2}-1\right)+\cdots+\left(L_{d+1}-1\right)+1$. Let $M(d, k, l)$ be the Ramsey number determined by Lemma 5.2. Similar to what was done in Theorem 6.4, we recursively construct the following sequence of finite numbers: let $K_{1}=M(d, 1, L)=N_{1}$; and for $i \geq 2$, let $M_{i}=\sum_{l=0}^{K_{i-1}}\left(\begin{array}{c}l+i-2 \\ i-2\end{array}\right), N_{i}=M\left(d, 2 K_{i-1}, M_{i}(L-1)+1\right)$ and $K_{i}=2 K_{i-1}+N_{i}$. 
Our proof now proceeds along a very similar line of argument (with some modification at the end) as in Theorem 6.4. Use graded reverse lex monomial ordering with $y_{1}>y_{2}>\cdots>y_{d}$ and $T_{1}>T_{2}>\cdots>T_{d}$, and consider an arbitrary relation in $y_{1}, \ldots, y_{d}$ of degree $N$

$$
F\left(T_{1}, \ldots, T_{d}\right)=\sum_{|\boldsymbol{n}|=N} r_{\boldsymbol{n}} \boldsymbol{T}^{\boldsymbol{n}}=0 .
$$

As in Theorem 6.4, it suffices to show that for any $1 \leq j \leq d-1$, if $F$ contains a term $r_{\boldsymbol{m}} \boldsymbol{T}^{\boldsymbol{m}}$ (say $\boldsymbol{m}=\left(m_{1}, \ldots, m_{d}\right)$ ) with $\operatorname{ord}_{<j+1}\left(\boldsymbol{T}^{\boldsymbol{m}}\right)>K_{j}$ we can write

$$
F\left(T_{1}, \ldots, T_{d}\right)=H\left(T_{1}, \ldots, T_{d}\right) G\left(T_{1}, \ldots, T_{d}\right)+F^{\prime}\left(T_{1}, \ldots, T_{d}\right),
$$

where $H$ is a monomial divisible by $\prod_{i>j} T_{i}^{m_{i}}, G$ and $F^{\prime}$ both provide relations in $x_{1}, \ldots, x_{d}$, the leading term $r_{k} T^{k}$ of $G$ satisfies $r_{k} T^{k} \mid r_{m} T^{m}$, and further $\operatorname{ord}_{<j+1}\left(\boldsymbol{T}^{k}\right) \leq K_{j}$ and $\operatorname{ord}_{j+1}\left(\boldsymbol{T}^{k}\right)=\cdots=\operatorname{ord}_{d}\left(\boldsymbol{T}^{k}\right)=0$ (in particular, the degree of $G$ is bounded by $K_{j}$ ), and all terms $r_{n_{1}} T^{n_{1}}$ of $F^{\prime}$ with $\operatorname{ord}_{<j+1}\left(\boldsymbol{T}^{\boldsymbol{n}_{1}}\right)>K_{j}$ are smaller than $r_{m} T^{m}$.

Suppose our assertion is not true. As before, let $j$ be the smallest index for which there is a relation $F$ contradicting our assertion. Suppose $r_{n_{0}} \boldsymbol{T}^{n_{0}}$ is the largest term of $F$ for which ord ${ }_{<j+1}\left(\boldsymbol{T}^{\boldsymbol{n}_{0}}\right)>K_{j}$. We shall pick $F$ such that $r_{\boldsymbol{n}_{0}} \boldsymbol{T}^{\boldsymbol{n}_{0}}$ is smallest possible. Let $\boldsymbol{n}_{0}=\left(n_{01}, \ldots, n_{0 d}\right)$. For simplicity, we write $r$ for $r_{\boldsymbol{n}_{0}}$. We shall derive a contradiction.

From the choice of $j$, we may assume that $K=\operatorname{ord}_{<j}\left(\boldsymbol{T}^{n_{0}}\right) \leq K_{j-1}$. Again, we first observe that

$$
n_{0 j}>K_{j}-K_{j-1}=K_{j-1}+N_{j}
$$

Let $B=\left(y_{j+1}, \ldots, y_{d}\right)$. We proceed along the same line of argument used in going from (6-4) to (6-7), with the exception that instead of having $\mathscr{A}(R)$ we now have $\mathscr{A}(R)+\left(y_{d}\right)$ being $\mathfrak{m}$-primary. Hence, $y_{j}^{q_{j}^{\prime}}-c y_{d} \in \mathscr{A}(R)$ for some positive integer $q_{j}^{\prime}$ and $c \in R$, and so

$$
\left(y_{j}^{q_{j}^{\prime}}-c y_{d}\right)\left(r \frac{\boldsymbol{y}^{\boldsymbol{n}_{0}}}{\prod_{i>j} y_{i}^{n_{0 i}}}+\sum_{\substack{\boldsymbol{n} \in \boldsymbol{J}, \boldsymbol{n}<\boldsymbol{n}_{0}, \prod_{i>j} y_{i} \boldsymbol{n}_{i} \mid \boldsymbol{y}^{\boldsymbol{n}}}} r_{\boldsymbol{n}} \frac{\boldsymbol{y}^{\boldsymbol{n}}}{\prod_{i>j} y_{i}^{n_{0 i}}}\right) \in B .
$$

Since $y_{d} \in B$, we get an equality similar to (6-7):

$$
y_{j}^{q_{j}^{\prime}}\left(r \frac{\boldsymbol{y}^{\boldsymbol{n}_{0}}}{\prod_{i>j} y_{i}^{n_{0 i}}}+\sum_{\substack{\boldsymbol{n} \in \boldsymbol{J}, \boldsymbol{n}<\boldsymbol{n}_{0},\left.\prod_{i>j} y_{i}^{n_{0} i}\right|_{\boldsymbol{y}^{\boldsymbol{n}}}}} r_{\boldsymbol{n}} \frac{\boldsymbol{y}^{\boldsymbol{n}}}{\prod_{i>j} y_{i}^{n_{0 i}}}\right) \in B .
$$


By a similar argument as in Theorem 6.4 again (as to get (6-10) and the condition in the next paragraph), we obtain a new relation in $y_{1}, \ldots, y_{d}$ :

$$
\begin{aligned}
Q\left(T_{1}, \ldots, T_{d}\right) & =r T_{1}^{n_{01}} \ldots T_{j-1}^{n_{0(j-1)}} T_{j}^{n_{0 j}+q_{j}}+\sum_{\substack{|\boldsymbol{m}|=q_{j}+K+n_{0 j}, \boldsymbol{m}<\left(n_{01}, \ldots, n_{0 j}+q_{j}, 0, \ldots, 0\right)}} u_{\boldsymbol{m}} \boldsymbol{T}^{\boldsymbol{m}} \\
& =r \boldsymbol{T}^{\boldsymbol{m}_{0}}+\sum_{\boldsymbol{n}<\boldsymbol{m}_{0}} u_{\boldsymbol{m}} \boldsymbol{T}^{\boldsymbol{m}}, \quad
\end{aligned}
$$

where $\boldsymbol{m}_{0}=\left(m_{01}, \ldots, m_{0 d}\right)=\left(n_{01}, \ldots, n_{0(j-1)}, n_{0 j}+q_{j}, 0, \ldots, 0\right)$, and each term $u_{\boldsymbol{m}} \boldsymbol{T}^{\boldsymbol{m}}$ in $Q$ satisfies ord $\operatorname{orj}_{<j}\left(\boldsymbol{T}^{\boldsymbol{m}}\right) \leq K_{j-1}$.

Let $\gamma \in \mathscr{A}(R)$ be a homology multiplier in $R$ such that $\left(\gamma, y_{1}, \ldots, \widehat{y_{j}}, \ldots, y_{d}\right)$ is an s.o.p. We also pick $\gamma$ such that $y_{d}$ is not in any associated primes of $\gamma$. Since $\gamma$ is part of an s.o.p. and $H_{\mathfrak{m}}^{0}(R)=0, \gamma$ is not a zero-divisor. Recall that $K=\operatorname{ord}_{<j}\left(\boldsymbol{T}^{\boldsymbol{n}_{0}}\right)=\operatorname{ord}_{<j}\left(\boldsymbol{T}^{\boldsymbol{m}_{0}}\right) \leq K_{j-1}$. For each $1 \leq p \leq m_{0 j}-K_{j-1}+1$, let $F_{p}\left(T_{1}, \ldots, T_{d}\right)$ be the sum of all terms in $Q$ that are divisible by $T_{j}^{m_{0 j}-K_{j-1}-p+1}$, and let $G_{p}\left(T_{1}, \ldots, T_{d}\right)=\frac{F_{p}\left(T_{1}, \ldots, T_{d}\right)}{T_{j}^{m_{0 j}-K_{j-1}-p+1}}=r T_{1}^{m_{01}} \ldots T_{j-1}^{m_{0(j-1)}} T_{j}^{K_{j-1}+p-1}+$ smaller terms. We can continue in the same line of argument as in Theorem 6.4, up to (6-13), to get a system of equalities which is similar to (6-13):

$$
\begin{aligned}
\gamma G_{1} & =s_{1} \boldsymbol{y}^{\boldsymbol{m}_{1}}+\sum_{\boldsymbol{m}<\boldsymbol{m}_{1}} s_{\boldsymbol{m}} \boldsymbol{y}^{\boldsymbol{m}}, \\
\gamma G_{2} & =s_{2} \boldsymbol{y}^{\boldsymbol{m}_{2}}+\sum_{\boldsymbol{m}<\boldsymbol{m}_{2}} s_{\boldsymbol{m}} \boldsymbol{y}^{\boldsymbol{m}}, \quad \ldots, \\
\gamma G_{p} & =s_{p} \boldsymbol{y}^{\boldsymbol{m}_{p}}+\sum_{\boldsymbol{m}<\boldsymbol{m}_{p}} s_{\boldsymbol{m}} \boldsymbol{y}^{\boldsymbol{m}}, \quad \ldots, \\
\gamma G_{m_{0 j}-K_{j-1}+1} & =s_{m_{0 j}-K_{j-1}+1} \boldsymbol{y}^{\boldsymbol{m}_{m_{0 j}-K_{j-1}+1}}+\sum_{\boldsymbol{m}<\boldsymbol{m}_{m_{0 j}-K_{j-1}+1}} s_{\boldsymbol{m}} \boldsymbol{y}^{\boldsymbol{m}},
\end{aligned}
$$

where $\boldsymbol{y}_{p}^{\boldsymbol{m}} \in C_{p}=\left(y_{1}, \ldots, \widehat{y_{j}}, \ldots, y_{d}\right)^{K_{j-1}} B^{K+p},\left|\boldsymbol{m}_{p}\right|=K_{j-1}+K+p$, and $G_{p}$ denotes $G_{p}\left(y_{1}, \ldots, y_{d}\right)$, for $1 \leq p \leq m_{0 j}-K_{j-1}+1$. Let $H_{p}=H_{p}\left(y_{1}, \ldots, y_{d}\right)$ be the right-hand side of the $p$-th equality in (7-5). As before, among all possible system of the form (7-5) associated to the relation $Q\left(T_{1}, \ldots, T_{d}\right)$, we choose one such that all the leading terms on the right-hand side are minimal.

The following claim is similar to Claim 6.5.

Claim 7.3. There exists an integer $p \leq N_{j}$ such that $H_{p}=0$.

Proof. By (7-4), we have $m_{0 j}-K_{j-1}+1 \geq N_{j}+1$. By contradiction, suppose the assertion is false. That is, $s_{1}, \ldots, s_{N_{j}}$ are all nonzero. Fix an integer $1 \leq p \leq$ $m_{0 j}-K_{j-1}+1$, and suppose the $y_{i}$ 's that appear in $\boldsymbol{y}^{\boldsymbol{m}_{p}}$ are in $\left\{y_{i} \mid i \geq h\right\} \backslash\left\{y_{j}\right\}$ (and $h$ is chosen to be the largest integer with this property). 
If $h<d$, we have

$$
\begin{aligned}
s_{p} & \in\left(\gamma,\left\{\boldsymbol{y}^{\boldsymbol{m}} \in C_{p} \mid \boldsymbol{m}<\boldsymbol{m}_{p}\right\}\right): \boldsymbol{y}^{\boldsymbol{m}_{p}} \\
& =\left(\gamma,\left\{\boldsymbol{x}^{\boldsymbol{m}} \in C_{p} \mid \operatorname{ord}_{i}\left(\boldsymbol{y}^{\boldsymbol{m}}\right)>\operatorname{ord}_{i}\left(\boldsymbol{y}^{\boldsymbol{m}_{p}}\right) \text { for some } i \geq h+1\right\}\right): \boldsymbol{y}^{\boldsymbol{m}_{p}} .
\end{aligned}
$$

Choose $\alpha \in \mathscr{A}(R)$ such that the images of $\left(\gamma,\left\{y_{h+1}, \ldots, y_{d-1}\right\} \backslash\left\{y_{j}\right\}, \alpha\right)$ form a part of an s.o.p. in $R /\left(x_{d}^{\prime}\right)$ (this is possible because $h \neq j$ and $d \geq 3$ ). Then,

$$
\alpha s_{p} \in\left(\gamma,\left\{y_{h+1}, \ldots, y_{d}\right\} \backslash\left\{y_{j}\right\}\right) \subseteq\left(\gamma,\left\{y_{h+1}, \ldots, y_{d-1}\right\} \backslash\left\{y_{j}\right\}, x_{d}^{\prime}\right),
$$

i.e.,

$$
s_{p} \in\left(\gamma,\left\{y_{h+1}, \ldots, y_{d-1}\right\} \backslash\left\{y_{j}\right\}, x_{d}^{\prime}\right): \alpha .
$$

Let $\tilde{\mathfrak{m}}, \tilde{s_{p}}, \tilde{\gamma}, \tilde{y}_{i}(i=1, \ldots, d)$, and $\tilde{\alpha}$ be the images of $\mathfrak{m}, s_{p}, \gamma, y_{i}(i=1, \ldots, d)$, and $\alpha$ in $R /\left(x_{d}^{\prime}\right)$. Since $x_{d}^{\prime}$ is part of an s.o.p., $R /\left(x_{d}^{\prime}\right)$ is a generalized CohenMacaulay ring. Since $\left(\tilde{\gamma},\left\{\tilde{y}_{h+1}, \ldots, \tilde{y}_{d-1}\right\} \backslash\left\{\tilde{y}_{j}\right\}, \tilde{\alpha}\right)$ is part of an s.o.p., $\tilde{\alpha}$ avoids all associated primes of $\left(\tilde{\gamma},\left\{\tilde{y}_{h+1}, \ldots, \tilde{y}_{d-1}\right\} \backslash\left\{\tilde{y}_{j}\right\}\right)$ except possibly $\tilde{\mathfrak{m}}$. Thus, we have

$$
\tilde{s_{p}} \in H_{\tilde{\mathfrak{m}}}^{0}\left(R /\left(\tilde{\gamma},\left\{\tilde{y}_{h+1}, \ldots, \tilde{y}_{d-1}\right\} \backslash\left\{\tilde{y}_{j}\right\}\right)\right),
$$

whence

$$
\bar{s}_{p} \in H_{\mathfrak{m}}^{0}\left(R /\left(\gamma,\left\{y_{h+1}, \ldots, y_{d-1}\right\} \backslash\left\{y_{j}\right\}, x_{d}^{\prime}\right)\right),
$$

here $\bar{s}_{p}$ denotes the image of $s_{p}$ in $R /\left(\gamma,\left\{y_{h+1}, \ldots, y_{d-1}\right\} \backslash\left\{y_{j}\right\}, x_{d}^{\prime}\right)$.

If $h=d$, then the $p$-th equality of (7-5) is

$$
\gamma G_{p}=s_{p} y_{d}^{m_{p}},
$$

where $\boldsymbol{m}_{p}=\left(0, \ldots, 0, m_{p}\right)$. Since $\gamma$ was chosen such that $y_{d}$ is not in any associated primes of $\gamma$, we must have

$$
\bar{s}_{p} \in H_{\mathfrak{m}}^{0}(R /(\gamma))
$$

Consider the sequence $\left\{\boldsymbol{m}_{1}, \boldsymbol{m}_{2}, \ldots, \boldsymbol{m}_{N_{j}}\right\}$. Since

$$
N_{j}=M\left(d, 2 K_{j-1}, M_{j}(L-1)+1\right) \geq M\left(d, K_{j-1}+K, M_{j}(L-1)+1\right),
$$

it follows from Lemma 5.2 that there exists an increasing subsequence of length $V=M_{j}(L-1)+1, \boldsymbol{m}_{j_{1}} \preceq \boldsymbol{m}_{j_{2}} \preceq \cdots \preceq \boldsymbol{m}_{j_{V}}$, with $1 \leq j_{1}<j_{2}<\cdots<j_{V} \leq N_{j} \leq$ $m_{0 j}-K_{j-1}$. Since $C_{p}=\left(y_{1}, \ldots, \widehat{y_{j}}, \ldots, y_{d}\right)^{K_{j-1}} B^{K+p}$, we have $\operatorname{ord}_{<j}\left(\boldsymbol{y}^{\boldsymbol{m}_{p}}\right) \leq$ $K_{j-1}$ for any $1 \leq p \leq m_{0 j}-K_{j-1}+1$. Thus, since $M_{j}$ is the number of $(j-1)$ tuples of nonnegative integers whose sum is at most $K_{j-1}$, we can choose from the sequence $\boldsymbol{m}_{j_{1}} \preceq \boldsymbol{m}_{j_{2}} \preceq \cdots \preceq \boldsymbol{m}_{j_{V}}$ a subsequence of length $L, \boldsymbol{m}_{i_{1}} \preceq \boldsymbol{m}_{i_{2}} \preceq \cdots \preceq \boldsymbol{m}_{i_{L}}$ such that they have the same $j-1$ first components, i.e. for any $1 \leq a, b \leq L$ we have $\left(\operatorname{ord}_{1}\left(\boldsymbol{y}^{\boldsymbol{m}_{i a}}\right), \ldots, \operatorname{ord}_{j-1}\left(\boldsymbol{y}^{\boldsymbol{m}_{i a}}\right)\right)=\left(\operatorname{ord}_{1}\left(\boldsymbol{y}^{\boldsymbol{m}_{i_{b}}}\right), \ldots, \operatorname{ord}_{j-1}\left(\boldsymbol{y}^{\boldsymbol{m}_{i_{b}}}\right)\right)$. Since $L=\left(L_{2}-1\right)+\cdots+\left(L_{d+1}-1\right)+1$, we can choose from this sequence a subsequence 
$\boldsymbol{m}_{p_{1}} \preceq \boldsymbol{m}_{p_{2}} \preceq \cdots \preceq \boldsymbol{m}_{p_{L_{q}}}$, for some $2 \leq q \leq d+1$, with the following property: if $q \leq d$ then

$$
\bar{s}_{p_{t}} \in H_{\mathfrak{m}}^{0}\left(R /\left(\gamma,\left\{y_{q+1}, \ldots, y_{d-1}\right\} \backslash\left\{y_{j}\right\}, x_{d}^{\prime}\right)\right),
$$

for all $t=1, \ldots, L_{q}$, and if $q=d+1$ then $\bar{s}_{p_{t}} \in H_{\mathfrak{m}}^{0}(R /(\gamma))$ for all $t=1, \ldots, L_{q}$. Notice that when $q=d+1$, as in (7-6), for all $t=1, \ldots, L_{q}$, the $p_{t}$-th equality of $(7-5)$ is

$$
\gamma G_{p_{t}}=s_{p_{t}} y_{d}^{m_{p_{t}}}
$$

where $\boldsymbol{m}_{p_{t}}=\left(0, \ldots, 0, m_{p_{t}}\right)$.

If $q=d+1$, it follows from the choice of $L_{q}$ that we can write

$$
s_{p_{L_{q}}}=\sum_{i=1}^{L_{q}-1} b_{i} s_{p_{i}}+a \gamma .
$$

Substituting this into the equality $\gamma G_{p_{L_{q}}}=H_{p_{L_{q}}}$, we get

$$
\gamma\left(G_{p_{L_{q}}}-a y_{d}^{m_{p_{L_{q}}}}\right)=\sum_{i=1}^{L_{q}-1}\left(b_{i} y_{d}^{m_{p_{L_{q}}}-m_{p_{i}}}\right) s_{p_{i}} y_{d}^{m_{p_{i}}} .
$$

We can now use the $p_{1}$-th, $\ldots, p_{L_{q}-1}$-th equalities in (7-5) to simplify the righthand side of (7-7) as we did in the 2-dimensional case (Theorem 6.1), bringing all terms with $\gamma$ to the left-hand side and absorbing extra powers into the coefficient if necessary, to get

$$
\gamma\left(G_{p_{L_{q}}}+\text { terms smaller than } r y_{1}^{m_{01}} \ldots y_{j-1}^{m_{0(j-1)}} y_{j}^{K_{j-1}+p_{L_{q}}-1}\right)=0 .
$$

This contradicts the fact that the right-hand side of (7-5) was chosen to be minimal.

Consider the case $q \leq d$. By the choice of $L_{q}$, we can write

$$
s_{p_{L_{q}}}=\sum_{\substack{q<i<d \\ i \neq j}} a_{i} y_{i}+a_{j} \gamma+a_{d} x_{d}^{\prime}+\sum_{i=1}^{L_{q}-1} b_{i} s_{p_{i}} .
$$

Substituting this into the equality $\gamma G_{p_{L_{q}}}=H_{p_{L_{q}}}$, we get

(7-8) $\quad \gamma\left(G_{p_{L_{q}}}-a_{j} \boldsymbol{y}^{\boldsymbol{m}_{p_{L_{q}}}}\right)$

$$
\begin{aligned}
& =\sum_{\substack{q<i<d, i \neq j}} a_{i} y_{i} \boldsymbol{y}^{\boldsymbol{m}_{p_{L_{q}}}}+a_{d} x_{d}^{\prime} \boldsymbol{y}^{\boldsymbol{m}_{p_{L_{q}}}}+\sum_{i=1}^{L_{q}-1} b_{i} s_{p_{i}} \boldsymbol{y}^{\boldsymbol{m}_{p_{L_{q}}}}+\sum_{\boldsymbol{m}<\boldsymbol{m}_{p_{L_{q}}}} s_{\boldsymbol{m}} \boldsymbol{y}^{\boldsymbol{m}} \\
& =\sum_{\substack{q<i<d \\
i \neq j}}\left(a_{i} y_{q}\right) \frac{\boldsymbol{y}^{\boldsymbol{m}_{p_{L_{q}}}} y_{i}}{y_{q}}+\sum_{i=1}^{L_{q}-1}\left(b_{i} \frac{\boldsymbol{y}^{\boldsymbol{m}_{p_{L_{q}}}}}{\boldsymbol{y}^{\boldsymbol{m}_{p_{i}}}}\right) s_{p_{i}} \boldsymbol{y}^{\boldsymbol{m}_{p_{i}}}+a_{d} x_{d}^{\prime} \boldsymbol{y}^{\boldsymbol{m}_{p_{L_{q}}}}+\sum_{\boldsymbol{m}<\boldsymbol{m}_{p_{L_{q}}}} s_{\boldsymbol{m}} \boldsymbol{y}^{\boldsymbol{m}} .
\end{aligned}
$$


Since $\boldsymbol{y}^{\boldsymbol{m}_{p_{L_{q}}}} \in C_{p_{L_{q}}}$, we obtain $a_{j} \boldsymbol{y}^{\boldsymbol{m}_{p_{L_{q}}}}<r y_{1}^{m_{01}} \ldots y_{j-1}^{m_{0(j-1)}} y_{j}^{K_{j-1}+p_{L_{q}}-1}$ (after absorbing extra powers into the coefficient if necessary). Also,

$$
\frac{\boldsymbol{y}^{\boldsymbol{m}_{p_{L_{q}}}} y_{i}}{y_{q}}<\boldsymbol{y}^{\boldsymbol{m}_{p_{L_{q}}}} \quad \text { for } \quad q<i<d .
$$

Since $\boldsymbol{y}^{\boldsymbol{m}_{p_{L_{q}}}}$ and $\boldsymbol{y}^{\boldsymbol{m}_{p_{i}}}$ have the same $j-1$ first components, we have $\boldsymbol{y}^{\boldsymbol{m}_{p_{L_{q}}}} / \boldsymbol{y}^{\boldsymbol{m}_{p_{i}}} \in$ $\left(y_{j+1}, \ldots, y_{d}\right)$, which then implies that all terms of $b_{i}\left(\boldsymbol{y}^{\boldsymbol{m}_{p_{L_{q}}}} / \boldsymbol{y}^{\boldsymbol{m}_{p_{i}}}\right) G_{p_{i}}$ are smaller than $r y_{1}^{m_{01}} \ldots y_{j-1}^{m_{0(j-1)}} y_{j}^{K_{j-1}+p_{L_{q}}-1}$. Thus, after using (7-5) to replace $s_{p_{i}} \boldsymbol{y}^{\boldsymbol{m}_{p_{i}}}$ by $\left[\gamma G_{p_{i}}-\sum_{|\boldsymbol{m}|=\left|\boldsymbol{m}_{p_{i}}\right|, \boldsymbol{m}<\boldsymbol{m}_{p_{i}}} s_{\boldsymbol{m}} \boldsymbol{y}^{\boldsymbol{m}}\right]$ in (7-8), we get a relation

(7-9) $\quad \gamma\left(G_{p_{L_{q}}}+\right.$ terms smaller than $\left.r y_{1}^{m_{01}} \ldots y_{j-1}^{m_{0(j-1)}} y_{j}^{K_{j-1}+p_{L_{q}-1}}\right)$

$$
=H_{p_{L q}}^{\prime}+a_{d} x_{d}^{\prime} \boldsymbol{y}^{\boldsymbol{m}_{p_{L_{q}}}}
$$

where the leading term of $H_{p_{L_{q}}}^{\prime}$ is strictly smaller than $s_{p_{L_{q}}} x^{m_{p_{L_{q}}}}$, which is the leading term of $H_{p_{L_{q}}}$.

If $x_{d}^{\prime}=x_{d}$ (that is, $x_{d}$ is in a small power of $\mathfrak{m}$ ), then

$$
a_{d} x_{d}^{\prime} \boldsymbol{y}^{\boldsymbol{m}_{p_{L_{q}}}}=\left(a_{d} y_{q}\right) \frac{\boldsymbol{y}^{\boldsymbol{m}_{p_{L_{q}}}} x_{d}^{\prime}}{y_{q}} .
$$

Thus, by absorbing $y_{q}$ into the coefficient, $a_{d} x_{d}^{\prime} y^{m_{p_{L_{q}}}}$ gives a term strictly smaller than $\boldsymbol{y}^{\boldsymbol{m}_{p_{L_{q}}}}$. We can rename $H_{p_{L_{q}}}^{\prime}+a_{d} x_{d}^{\prime} \boldsymbol{y}^{\boldsymbol{m}_{p_{L_{q}}}}$ in (7-9) as $H_{p_{L_{q}}}^{\prime}$. This contradicts the way (7-5) was chosen.

Suppose now that $y_{d}=x_{d}^{\prime} w^{t}$. Write $y_{q}=x_{q}^{\prime} w^{t}+\beta$ for some $\beta \in \mathscr{A}(R)$ (this is possible since $t_{x_{q}} \geq t_{x_{d}}$ ). Write $\boldsymbol{y}^{\boldsymbol{m}_{p_{L_{q}}}}=\prod_{i \geq q, i \neq j} y_{i}^{l_{i}}$. Then, since all terms of $H_{p_{L_{q}}}^{\prime}$ are smaller than $\boldsymbol{y}^{\boldsymbol{m}_{p_{L_{q}}}}$, it follows from (7-9) that

$$
\begin{aligned}
a_{d} & \in\left(\gamma,\left\{y_{i}^{l_{i}+1} \mid i>q, i \neq j\right\}\right): x_{d}^{\prime} \boldsymbol{y}^{\boldsymbol{m}_{p_{L_{q}}}} \\
& \subseteq\left(\gamma,\left\{y_{i}^{l_{i}+1} \mid q<i<d, i \neq j\right\},\left(w^{t}\right)^{l_{d}+1}\right): x_{d}^{\prime} \boldsymbol{y}^{\boldsymbol{m}_{p_{L_{q}}}} .
\end{aligned}
$$

Therefore,

$$
\beta a_{d} \in\left(\gamma,\left\{y_{i} \mid q<i<d, i \neq j\right\}, w^{t}\right) .
$$

We also have

$$
a_{d} x_{d}^{\prime} \boldsymbol{y}^{\boldsymbol{m}_{p_{L_{q}}}}=a_{d} x_{d}^{\prime}\left(x_{q}^{\prime} w^{t}+\beta\right) y_{q}^{l_{q}-1}\left(\prod_{\substack{q<i<d \\ i \neq j}} y_{i}^{l_{i}}\right)\left(x_{d}^{\prime} w^{t}\right)^{l_{d}}=
$$




$$
\begin{aligned}
& =a_{d} x_{d}^{\prime} x_{q}^{\prime} w^{t} y_{q}^{l_{q}-1}\left(\prod_{\substack{q<i<d \\
i \neq j}} y_{i}^{l_{i}}\right)\left(x_{d}^{\prime} w^{t}\right)^{l_{d}}+\beta a_{d} x_{d}^{\prime} y_{q}^{l_{q}-1}\left(\prod_{\substack{q<i<d \\
i \neq j}} y_{i}^{l_{i}}\right)\left(x_{d}^{\prime} w^{t}\right)^{l_{d}} \\
& =\left(a_{d} x_{q}^{\prime}\right)\left(x_{d}^{\prime} w^{t}\right)^{l_{d}+1} y_{q}^{l_{q}-1}\left(\prod_{\substack{q<i<d \\
i \neq j}} y_{i}^{l_{i}}\right)+\beta a_{d} x_{d}^{\prime} y_{q}^{l_{q}-1}\left(\prod_{\substack{q<i<d \\
i \neq j}} y_{i}^{l_{i}}\right)\left(x_{d}^{\prime} w^{t}\right)^{l_{d}} \\
& =\left(a_{d} x_{q}^{\prime}\right) y_{q}^{l_{q}-1}\left(\prod_{\substack{q<i<d \\
i \neq j}} y_{i}^{l_{i}}\right) y_{d}^{l_{d}+1}+\beta a_{d} x_{d}^{\prime} y_{q}^{l_{q}-1}\left(\prod_{\substack{q<i<d \\
i \neq j}} y_{i}^{l_{i}}\right)\left(x_{d}^{\prime} w^{t^{\prime}}\right)^{l_{d}} .
\end{aligned}
$$

By $(7-10)$, we can write

$$
\beta a_{d}=c_{j} \gamma+\sum_{\substack{q<u<d \\ i \neq j}} c_{u} y_{u}+c_{d} w^{t}
$$

Substituting this into (7-11), we get

$(7-12) \quad a_{d} x_{d}^{\prime} \boldsymbol{y}^{\boldsymbol{m}_{p_{L_{q}}}}$

$$
\begin{aligned}
& =\left(a_{d} x_{q}^{\prime}\right) y_{q}^{l_{q}-1}\left(\prod_{\substack{q<i<d \\
i \neq j}} y_{i}^{l_{i}}\right) y_{d}^{l_{d}+1}+c_{j} \gamma x_{d}^{\prime} y_{q}^{l_{q}-1}\left(\prod_{\substack{q<i<d \\
i \neq j}} y_{i}^{l_{i}}\right)\left(x_{d}^{\prime} w^{t}\right)^{l_{d}} \\
& +\sum_{\substack{q<u<d \\
i \neq j}}\left(c_{u} x_{d}^{\prime}\right) y_{u} y_{q}^{l_{q}-1}\left(\prod_{\substack{q<i<d \\
i \neq j}} y_{i}^{l_{i}}\right)\left(x_{d}^{\prime} w^{t}\right)^{l_{d}} \\
& +c_{d}\left(x_{d}^{\prime} w^{t}\right) y_{q}^{l_{q}-1}\left(\prod_{\substack{q<i<d \\
i \neq j}} y_{i}^{l_{i}}\right)\left(x_{d}^{\prime} w^{t}\right)^{l_{d}} \\
& =\left(a_{d} x_{q}^{\prime}\right) y_{q}^{l_{q}-1}\left(\prod_{\substack{q<i<d \\
i \neq j}} y_{i}^{l_{i}}\right) y_{d}^{l_{d}+1}+c_{j} \gamma x_{d}^{\prime} y_{q}^{l_{q}-1}\left(\prod_{\substack{q<i<d \\
i \neq j}} y_{i}^{l_{i}}\right) y_{d}^{l_{d}} \\
& +\sum_{\substack{q<u<d \\
i \neq j}}\left(c_{u} x_{d}^{\prime}\right) y_{u} y_{q}^{l_{q}-1}\left(\prod_{\substack{q<i<d \\
i \neq j}} y_{i}^{l_{i}}\right) y_{d}^{l_{d}}+c_{d} y_{q}^{l_{q}-1}\left(\prod_{\substack{q<i<d \\
i \neq j}} y_{i}^{l_{i}}\right) y_{d}^{l_{d}+1} \text {. }
\end{aligned}
$$

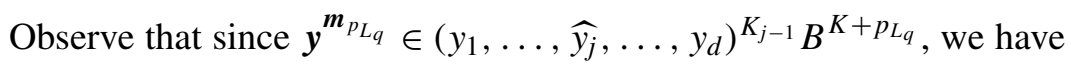

$$
y_{q}^{l_{q}-1}\left(\prod_{\substack{q<i<d \\ i \neq j}} y_{i}^{l_{i}}\right) y_{d}^{l_{d}}=\frac{\boldsymbol{y}^{\boldsymbol{m}_{p_{L_{q}}}}}{y_{q}} \in\left(y_{1}, \ldots, \widehat{y_{j}}, \ldots, y_{d}\right)^{K_{j-1}} B^{K+p_{L_{q}}-1}
$$

that is,

$$
\left(c_{j} x_{d}^{\prime}\right) y_{q}^{l_{q}-1}\left(\prod_{\substack{q<i<d \\ i \neq j}} y_{i}^{l_{i}}\right) y_{d}^{l_{d}}<r y_{1}^{m_{01}} \ldots y_{j-1}^{m_{0(j-1)}} y_{j}^{K_{j-1}+p_{L_{q}}-1}
$$


Hence, substituting (7-12) into (7-9), bringing terms with $\gamma$ to the left-hand side, we get

$$
\gamma\left(G_{p_{L_{q}}}+\text { terms smaller than } r y_{1}^{m_{01}} \ldots y_{j-1}^{m_{0(j-1)}} y_{j}^{K_{j-1}+p_{L_{q}}-1}\right)=H_{p_{L_{q}}}^{\prime \prime},
$$

where the leading terms of $H_{p_{L_{q}}}^{\prime \prime}$ is strictly smaller than $\boldsymbol{y}^{\boldsymbol{m}_{p_{L_{q}}}}$ which is the leading term of $H_{p_{L_{q}}}$. This contradicts the way (7-5) was chosen. Our claim is proved.

We have just shown that there must exist an integer $p \leq N_{j}$ such that

$$
\gamma G_{p}=0 .
$$

Thus, since $\gamma$ is not a zero-divisor, we have $G_{p}=0$. That is,

$$
r y_{1}^{m_{01}} \ldots y_{j-1}^{m_{0(j-1)}} y_{j}^{K_{j-1}+p-1}+\text { smaller terms }=0 .
$$

This gives a new relation in $y_{1}, \ldots, y_{d}$, which by abusing language we also denote by $G_{p}$,

$$
\begin{aligned}
G_{p}\left(T_{1}, \ldots, T_{d}\right) & =r T_{1}^{m_{01}} \ldots T_{j-1}^{m_{0(j-1)}} T_{j}^{K_{j-1}+p-1}+\text { smaller terms } \\
& =r T_{1}^{n_{01}} \ldots T_{j-1}^{n_{0(j-1)}} T_{j}^{K_{j-1}+p-1}+\text { smaller terms. }
\end{aligned}
$$

Once again, observe that $K_{j-1}+p-1 \leq K_{j-1}+N_{j}-1<n_{0 j}$ by (7-4). Therefore, we can write

$$
F\left(T_{1}, \ldots, T_{d}\right)=\left[T_{j}^{n_{0 j}-K_{j-1}-p+1} \prod_{i>j} T_{i}^{n_{0 i}}\right] G_{p}\left(T_{1}, \ldots, T_{d}\right)+F^{\prime}\left(T_{1}, \ldots, T_{d}\right),
$$

where $G_{p}\left(T_{1}, \ldots, T_{d}\right)$ and $F^{\prime}\left(T_{1}, \ldots, T_{d}\right)$ are relations in $y_{1}, \ldots, y_{d}$. Again, it is clear that every term $r_{\boldsymbol{n}} \boldsymbol{T}^{\boldsymbol{n}}$ of $F^{\prime}$ with ord ${ }_{<j+1}\left(\boldsymbol{T}^{\boldsymbol{n}}\right)>K_{j}$ is smaller than $r_{\boldsymbol{n}_{0}} \boldsymbol{T}^{\boldsymbol{n}_{0}}$. We obtain a contradiction. Hence, the theorem is proved.

Let $R$ be a ring of positive prime characteristic $p$. We denote the $e$ th power of the Frobenius endomorphism $f: R \rightarrow R$ sending $x \mapsto x^{p}$ by $f^{e}$. For $q=p^{e}$, a power of $p$, and $I \subseteq R$ we let $I^{[q]}=\left(i^{q} \mid i \in I\right)$. The ideal $I \subseteq R$ is called Frobenius closed if whenever $x^{q} \in I^{[q]}$ then $x \in I$. The ring $R$ is called $F$-pure if $f$ is a pure morphism and cyclically $F$-pure if all ideals of $R$ are Frobenius closed. When $R$ is excellent these conditions are equivalent [Hochster 1977].

Also, when $R=S / J$ is the image of a regular local ring $(S, \mathfrak{n})$, Fedder [1983] has given a criterion for $F$-purity in terms of $J$ : namely, $R$ is $F$-pure if and only if $J^{[p]}:_{S} J \nsubseteq \mathfrak{n}^{[p]}$.

When $(R, \mathfrak{m})$ is an excellent local ring then $R$ is $F$-pure if and only if $\widehat{R}$ is $F$ pure. Moreover, in an $F$-pure ring, the ideal $\mathscr{A}(R)$ is radical. We may thus apply Theorem 7.2 to obtain: 
Corollary 7.4. Let $(R, \mathfrak{m})$ be an complete local equidimensional $F$-pure ring such that the defining ideal of the non-CM locus is a dimension one prime ideal. Then $R$ has a uniform bound on relation type of parameter ideals.

\section{References}

[Aberbach and Huneke 1993] I. M. Aberbach and C. Huneke, "An improved Briançon-Skoda theorem with applications to the Cohen-Macaulayness of Rees algebras", Math. Ann. 297:2 (1993), 343-369. MR 95b:13005 Zbl 0788.13001

[Adhikari 2002] S. D. Adhikari, Aspects of combinatorics and combinatorial number theory, Narosa, New Delhi, and CRC Press, Boca Raton (FL), 2002. MR 2003d:05001 Zbl 0998.05065

[Duncan and O'Carroll 1989] A. J. Duncan and L. O'Carroll, "A full uniform Artin-Rees theorem", J. Reine Angew. Math. 394 (1989), 203-207. MR 90c:13011 Zbl 0659.13002

[Eliahou and Kervaire 1990] S. Eliahou and M. Kervaire, "Minimal resolutions of some monomial ideals”, J. Algebra 129:1 (1990), 1-25. MR 91b:13019 Zbl 0701.13006

[Fedder 1983] R. Fedder, “F-purity and rational singularity”, Trans. Amer. Math. Soc. 278:2 (1983), 461-480. MR 84h:13031 Zbl 0519.13017

[Hochster 1977] M. Hochster, "Cyclic purity versus purity in excellent Noetherian rings", Trans. Amer. Math. Soc. 231:2 (1977), 463-488. MR 57 \#3111 Zbl 0369.13005

[Hochster and Huneke 1990] M. Hochster and C. Huneke, "Tight closure, invariant theory, and the Briançon-Skoda theorem”, J. Amer. Math. Soc. 3 (1990), 31-116. MR 91g:13010 Zbl 0701.13002

[Hochster and Huneke 1992] M. Hochster and C. Huneke, "Infinite integral extensions and big Cohen-Macaulay algebras”, Ann. of Math. (2) 135:1 (1992), 53-89. MR 92m:13023 Zbl 0753. 13003

[Huneke 1980] C. Huneke, "On the symmetric and Rees algebra of an ideal generated by a $d$ sequence”, J. Algebra 62:2 (1980), 268-275. MR 81d:13016 Zbl 0439.13001

[Huneke 1992] C. Huneke, "Uniform bounds in Noetherian rings", Invent. Math. 107:1 (1992), 203223. MR 93b:13027 Zbl 0756.13001

[Lai 1995] Y.-H. Lai, “On the relation type of systems of parameters”, J. Algebra 175:1 (1995), 339-358. MR 96i:13026 Zbl 0846.13016

[O'Carroll 1987] L. O'Carroll, "A uniform Artin-Rees theorem and Zariski's main lemma on holomorphic functions”, Invent. Math. 90:3 (1987), 647-652. MR 89h:13013 Zbl 0611.14001

[O'Carroll 1991] L. O'Carroll, "A note on Artin-Rees numbers", Bull. London Math. Soc. 23:3 (1991), 209-212. MR 92i:13001a Zbl 0697.13002

[Planas-Vilanova 2000] F. Planas-Vilanova, "The strong uniform Artin-Rees property in codimension one”, J. Reine Angew. Math. 527 (2000), 185-201. MR 2001g:13051 Zbl 0988.13013

[Planas-Vilanova 2006] F. Planas-Vilanova, "An approach to the uniform Artin-Rees theorems from the notion of relation type", pp. 175-191 in Commutative algebra, edited by A. Corso et al., Lect. Notes Pure Appl. Math. 244, Taylor \& Francis, Boca Raton, FL, 2006. MR 2006h:13020 Zbl 02236744

[Raghavan 1991] K. N. Raghavan, Uniform annihilation of local cohomology and powers of ideals generated by quadratic sequences, Ph.D. thesis, Purdue University, West Lafayette, IN, 1991.

[Ramsey 1929] F. P. Ramsey, "On a problem of formal logic", Proc. London Math. Soc. (2) 30 (1929), 264-285. JFM 55.0032.04 
[Rees 1988] D. Rees, Lectures on the asymptotic theory of ideals, London Mathematical Society Lecture Note Series 113, Cambridge University Press, Cambridge, 1988. MR 90d:13012 Zbl 0669.13001

[Trung 1987] N. V. Trung, "Reduction exponent and degree bound for the defining equations of graded rings", Proc. Amer. Math. Soc. 101:2 (1987), 229-236. MR 89i:13031 Zbl 0641.13016

[Valla 1980] G. Valla, "On the symmetric and Rees algebras of an ideal", Manuscripta Math. 30:3 (1980), 239-255. MR 83b:14017 Zbl 0439.13002

[Wang 1997a] H.-J. Wang, "The relation-type conjecture holds for rings with finite local cohomology”, Comm. Algebra 25:3 (1997), 785-801. MR 98d:13017 Zbl 0885.13012

[Wang 1997b] H.-J. Wang, "Some uniform properties of 2-dimensional local rings", J. Algebra 188:1 (1997), 1-15. MR 98e:13006 Zbl 0889.13003

Received September 23, 2004. Revised January 25, 2005.

IAN M. ABERBACH

MATHEMATICS DEPARTMENT

UNIVERSITY OF MISSOURI

COLUMBia, MO 65211

UNITED STATES

aberbach@math.missouri.edu

http://www.math.missouri.edu/people/iaberbach.html

LAURA GHEZZI

MATHEMATICS DEPARTMENT

FLORIDA INTERNATIONAL UNIVERSITY

UNIVERSITY PARK, MIAMI FL 33199

UNITED STATES

ghezzil@ fiu.edu

http://www.fiu.edu/ ghezzil/

HUY TÀI HÀ

TULANE UNIVERSITY

DEPARTMENT OF MATHEMATICS

6823 St. Charles AVE.

NEW ORLEANS, LA 70118

UNITED STATES

tai@math.tulane.edu

http://www.math.tulane.edu/ tai/ 